\title{
Inter- and intramolecular glycosylation of exo-glycals promoted by metallic Lewis acids
}

\author{
Sheng-Jie Hsu, ${ }^{\text {a,b }}$ Hui-Chang Lin $^{\mathrm{a}}$ and Chun-Hung Lin ${ }^{\mathrm{a}, \mathrm{b}, *}$ \\ ${ }^{a}$ Institute of Biological Chemistry and Genomics Research Center, Academia Sinica, No. 128 Academia Road Section 2, \\ Nan-Kang, Taipei 11529, Taiwan \\ ${ }^{\mathrm{b}}$ Institute of Biochemical Sciences, College of Life Science, National Taiwan University, Taipei 10617, Taiwan \\ Received 2 February 2006; received in revised form 18 March 2006; accepted 1 April 2006 \\ Available online 2 May 2006
}

\begin{abstract}
Glycosyl carbonates were employed for inter- and intramolecular glycosylation reactions. A number of metallic Lewis Acids and solvents were examined to enhance the reactivity. The optimum conditions were found to be the use of $\mathrm{AlCl}_{3}$ in 1-nitropropane. The method was demonstrated to be useful for the intermolecular glycosyl transfer of several nucleophiles, including simple alcohols, sugars, and amino acid derivatives; however, intramolecular glycosylations were not successful.

(C) 2006 Elsevier Ltd. All rights reserved.
\end{abstract}

Keywords: Carbonate; exo-Glycal; Glycosylation; Lewis acid; Stereoselectivity

\section{Introduction}

C-glycosylidenes, often referred to as exo-glycals, are recognized as versatile key intermediates for the preparation of C-glycosides when the double bond is properly functionalized with high stereocontrol. Since the first report on exo-glycals appeared thirty years ago, ${ }^{1}$ extensive studies have been carried out with two major achievements in recent years. The first were practical methods to prepare exo-glycals with a wide range of double bond substitutions. $^{2-4}$ The second was to successfully apply exo-glycals to the synthesis of biologically important molecules, such as anti-tumor natural products and enzyme inhibitors. ${ }^{5}$ These advances have greatly expanded the availability of these molecules and their uses.

Analogous to the reactions of endo-glycals, exo-glycals have been shown to be reactive glycosyl donors. ${ }^{6}$ We previously reported that glycosylation of exo-glycals is applicable to various acceptors with exclusive $\alpha$-stereo- selectivity. The reactivity can be greatly enhanced by Ferrier-type rearrangement ${ }^{7}$ or/and microwave irra-

\footnotetext{
*Corresponding author. Tel.: +886 2 27890110; fax: +886 2 26514705; e-mail: chunhung@gate.sinica.edu.tw
}

diation. ${ }^{8}$ In addition, different reaction routes were found for the acid-catalyzed glycosylation of exo- and endo-glycals, where the reaction pathways of rearrangement and protonation were found to compete during the glycosylation reactions. Interestingly, exo-glycals have a favored pathway different from their endo-glycal counterparts. ${ }^{9}$ As part of our continuing efforts in the research of glycals, we herein report inter- and intramolecular glycosylation of exo-glycals. Optimization of the reaction conditions showed that the reaction proceeded best when done in 1-nitropropane and when the Lewis acid is $\mathrm{AlCl}_{3}$.

\section{Results and discussion}

\subsection{Effects of solvent and Lewis acid on glycosylation}

In our previous report, exo-glycal carbonate 1 was shown to be an efficient glycosyl donor using microwave-assisted glycosylation. ${ }^{8}$ The same reactions took a much longer time and gave lower yields with conventional heating (e.g., in DMF at reflux). The addition of Lewis acids, such as $\mathrm{InCl}_{3}$ or $\mathrm{AlCl}_{3}$, significantly improved the yields and reduced the reaction time, which prompted further 
Table 1. Glycosylation of exo-glycal 1 in various solvents ${ }^{\mathrm{a}}$

\begin{tabular}{|c|c|c|c|}
\hline Solvent & Time $(\mathrm{h})$ & Temperature $\left({ }^{\circ} \mathrm{C}\right)$ & Yield $(\%)$ \\
\hline 1-Nitropropane & 0.67 & 0 & 80 \\
\hline 1-Nitroethane & 0.67 & 0 & 74 \\
\hline Nitromethane & 0.67 & 0 & 75 \\
\hline $\mathrm{CH}_{2} \mathrm{Cl}_{2}$ & 0.67 & 0 & 68 \\
\hline $\mathrm{DMF}$ & 36 & 25 & ${ }^{\mathrm{b}}$ \\
\hline THF & 40 & 25 & $20^{\mathrm{c}}$ \\
\hline Toluene & 48 & 25 & $-^{\mathrm{b}}$ \\
\hline $\mathrm{CH}_{3} \mathrm{CN}$ & 96 & 25 & $21^{\mathrm{c}}$ \\
\hline
\end{tabular}

${ }^{a}$ All reactions were carried out in the presence of $\mathrm{AlCl}_{3}$ (1.0 equiv) and molecular sieves $(4 \AA)$. The donor and acceptor were present in a ratio of $1: 1$.

${ }^{\mathrm{b}}$ No reaction occurred.

${ }^{\mathrm{c}}$ About a half of the starting material still remained according to TLC.

investigations. Table 1 shows the glycosylation of 1,2:3,4$\mathrm{di}-O$-isopropylidene- $\alpha$-galactopyranose by $\mathbf{1}$ in various solvents. The reactions in DMF and toluene did not take place at $25^{\circ} \mathrm{C}$ although they indeed occurred at higher temperatures (e.g., at reflux). Good yields were obtained when nitroalkanes or dichloromethane was used as the solvent. We suggest that solvent polarity and basicity are two main factors playing a role. Because these reactions occur via an oxacarbonium ion intermediate, solvents of high polarity will help to stabilize the intermediate and thus enhance the reactivity. ${ }^{10}$ The enthalpic changes of complexation to boron trifluoride are dependent on the solvent and appear in the order: DMF > THF $>\mathrm{CH}_{3} \mathrm{CN}>\mathrm{CH}_{3} \mathrm{NO}_{2}>\mathrm{CH}_{2} \mathrm{Cl}_{2}{ }^{11,12}$ This analysis is in agreement with our observation that the top three solvents relatively stabilize Lewis acids and thus the resulting reactions either do not proceed at all, or proceed very slowly. Nitroalkanes have low coordinating ability probably due to resonance and we selected 1-nitropropane for further studies because this solvent afforded the best yield $(80 \%)$.

Fifteen metallic Lewis acids were then screened as activators. If the reactions did not proceed or were very slow, they were kept initially at $0{ }^{\circ} \mathrm{C}$ for $1.5 \mathrm{~h}$ and the temperature was raised to $25^{\circ} \mathrm{C}$ to finish the reaction. As shown in Table 2, the majority of reactions gave the desired products in good yields. The reactions appear to not have preference for the metal because Lewis acids derived from alkaline earth metals (Group IIA), Group IIIB, transition metals, and lanthanides are all able to promote the reaction, in comparison with the reaction without a Lewis acid. In particular, the reactions assisted by $\mathrm{AlCl}_{3}, \mathrm{SnCl}_{4}, \mathrm{Yb}(\mathrm{OTf})_{3}$, and $\mathrm{Cu}(\mathrm{OTf})_{2}$ were complete within an hour to provide $70-80 \%$ yields. Taking into account reagent cost, availability, and compatibility with protecting groups, $\mathrm{AlCl}_{3}$ appeared to be the best choice for additional investigations.

\subsection{Inter- and intramolecular glycosylations}

Using the optimized conditions, exo-glycosyl carbonates $\mathbf{1}$ and $\mathbf{2}$ were subjected to glycosylation with several alcohol nucleophiles, including simple alcohols, sugars, and amino acid derivatives (Table 3 ) to give glycoside products 3-12. Most of the reactions were complete in $40 \mathrm{~min}$ and gave moderate to good yields, except for more hindered substrates (entries iv and ix). The reactions displayed exclusive $\alpha$-stereoselectivity; that is, all the nucleophiles attacked from the bottom face of the sugar ring. This result is consistent with the anomeric effect because the $\alpha$-glycoside products are more thermodynamically stable. The product structure and stereochemistry were rigorously determined using DEPT, NOESY, and other spectroscopic data, in agreement with previous reports. ${ }^{6-9,13}$

The use of glycosyl carbonates as donors in glycosylation reactions ${ }^{14}$ was first reported by Mukaiyama and co-workers, where either dimethyl dichlorosilane-silver

Table 2. Effect of Lewis acid on the glycosylation of $\mathbf{1}$ in 1-nitropropane ${ }^{\mathrm{a}}$

\begin{tabular}{llllllll}
\hline Lewis acid & Time $(\mathrm{h})$ & Temperature $\left({ }^{\circ} \mathrm{C}\right)$ & Yield $(\%)$ & Lewis acid & Time $(\mathrm{h})$ & Temperature $\left({ }^{\circ} \mathrm{C}\right)$ & Yield $(\%)$ \\
\hline $\mathrm{AlCl}_{3}$ & 0.67 & 0 & 80 & $\mathrm{Mg}\left(\mathrm{ClO}_{4}\right)_{2}$ & $1.5,1^{\mathrm{b}}$ & 0,25 & 79 \\
$\mathrm{InCl}_{3}$ & $1.5,6^{\mathrm{b}}$ & 0,25 & 82 & $\mathrm{Mn}\left(\mathrm{CH}_{3} \mathrm{CO}_{2}\right)_{2}$ & $1.5,28^{\mathrm{b}}$ & 0,25 & 0 \\
$\mathrm{ZnCl}_{2}$ & $1.5,1^{\mathrm{b}}$ & 0,25 & 75 & $\mathrm{Yb}(\mathrm{OTf})_{3}$ & 0.67 & 0 & 81 \\
$\mathrm{SnCl}_{4}$ & 0.33 & 0 & 70 & $\mathrm{La}(\mathrm{OTf})_{3}$ & $1.5,1.5^{\mathrm{b}}$ & 0,25 & 0 \\
$\mathrm{SnCl}_{2}$ & 2.5 & 0 & 75 & $\mathrm{Eu}(\mathrm{OTf})_{3}$ & 2 & 0 & 77 \\
$\mathrm{SmCl}_{3}$ & $1.5,20$ & 0,25 & 80 & $\mathrm{Zn}(\mathrm{OTf})_{2}$ & $1.5,1^{\mathrm{b}}$ & 0,25 & 72 \\
$\mathrm{FeCl}_{3}$ & $1.5,6^{\mathrm{b}}$ & 0,25 & 80 & $\mathrm{Cu}(\mathrm{OTf})_{2}$ & 0.67 & 0 & 71 \\
$\mathrm{CeCl}_{3}$ & 1.5, & 0,25 & $-{ }^{\mathrm{c}}$ & & & & \\
\hline
\end{tabular}

The reaction is the same as that shown in Table 1 except for the solvent and Lewis acid.

${ }^{a}$ All reactions were carried out in the presence of Lewis acid (1.0 equiv) in 1-nitropropane and molecular sieves (4 $\AA$ ). The donor and acceptor were present in a 1:1 ratio.

${ }^{\mathrm{b}}$ The reaction was initially kept at $0{ }^{\circ} \mathrm{C}$ for $1.5 \mathrm{~h}$ and the temperature was then raised to $25^{\circ} \mathrm{C}$ for the indicated period of time.

${ }^{\mathrm{c}}$ No reaction occurred. 
Table 3. Glycosylations of $\mathbf{1}$ and $\mathbf{2}$ with various alcohols ${ }^{\mathrm{a}}$

Entry

${ }^{\text {a }}$ All reactions were carried out in 1-nitropropane with alcohol nucleophiles in the presence of molecular sieves ( $4 \AA$ ). perchlorate or tetrakis(pentafluorophenyl)borate was used as the catalyst. ${ }^{15,16}$ Excellent $\beta$-stereoselectivity was reported in their studies. A modified version was developed by Ley et al. to employ 1-imidazolylcarbonylor 1-imidazolylthiocarbonyl glycosides as glycosyl donors. ${ }^{17,18}$ In the presence of zinc bromide or silver perchlorate, the reactions in diethyl ether led to good $\alpha$-stereoselectivity, while the use of dichloromethane resulted in an increase in $\beta$-glycosides. The reactions were promoted by chelation of the metal atom to the carbonyl oxygen (or thiocarbonyl sulfur) and N3 of the imidazolyl group. It is interesting that our glycosylation reactions of exo-glycals generate $\alpha$-stereoselectivity exclusively.

Encouraged by these successes, we then turned the focus to intramolecular glycosylation. Suitable substrates should contain a donor and an acceptor in one molecule, that is, the $t$-butoxy group of carbonates $\mathbf{1}$ or $\mathbf{2}$ is replaced with another alcohol. The preparation of exo-glycal carbonates 13-16 (Fig. 1 and Scheme 1) was straightforward. ${ }^{19}$ Compound $\mathbf{1 7}$, the precursor of 2, reacted with $n$-butyl chloroformate to generate $\mathbf{1 3}$ in $85 \%$ yield, as shown in Scheme 1. Compound $\mathbf{1 5}$ was derived in a similar way from benzyl chloroformate ( $82 \%$ yield). A two-step procedure is required for the synthesis of carbonates $\mathbf{1 4}$ and $\mathbf{1 6}$. The reaction of methyl 2,3,4-tri- $O$-methyl- $\alpha$-D-glucopyranoside with carbonyl diimidazole (CDI) with a catalytic amount of $\mathrm{KOH}$ afforded 18, which was then coupled with exoglycal 17, by treatment with sodium hydride, giving carbonate $\mathbf{1 6}$ in $88 \%$ overall yield. Compound $\mathbf{1 4}$ was prepared in an analogous manner in $82 \%$ overall yield.

Compounds $13-16$ were heated at $125^{\circ} \mathrm{C}$ in 1-nitropropane to perform the intramolecular glycosylation. The desired products were obtained in low yields (Table 4). The addition of $\mathrm{AlCl}_{3}$ did not increase the yields, nor did substitution with other Lewis acids (e.g., $\mathrm{BF}_{3} \cdot \mathrm{OEt}_{2}$, or TMSOTf in $\mathrm{CH}_{2} \mathrm{Cl}_{2}$ ). Of the product that was formed, again, the $\alpha$-glycosides were produced. Compound 21 (Fig. 1), which has a free hydroxyl group, as inspired by Ito's intramolecular aglycon delivery using a $p$-alkoxybenzyl group for tethering, ${ }^{20}$ was also

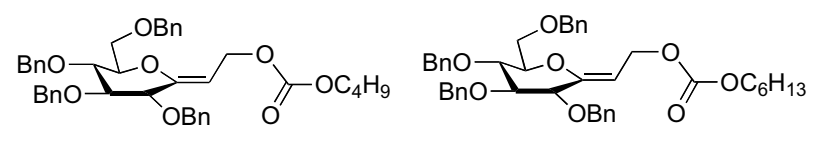

14
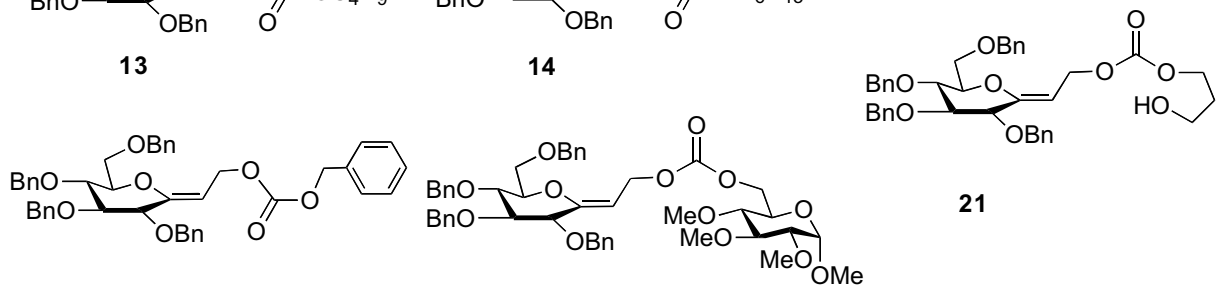

15

16

Figure 1. Structures of 13-16 and 21. 

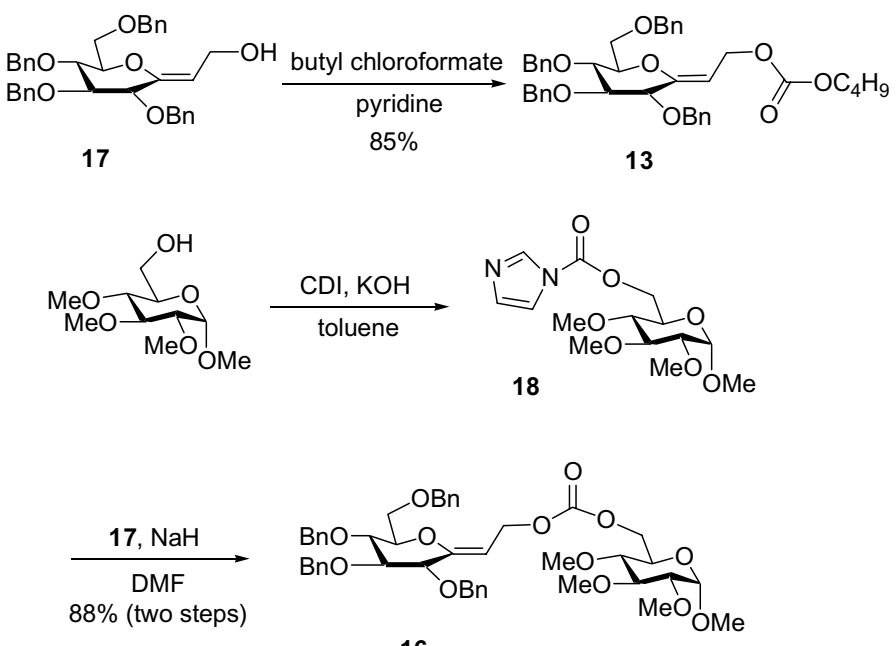

16

Scheme 1. Preparation of $\mathbf{1 3}$ and $\mathbf{1 6 .}$

Table 4. Intramolecular glycosylations of $\mathbf{1 3}-\mathbf{1 6}^{\mathrm{a}}$
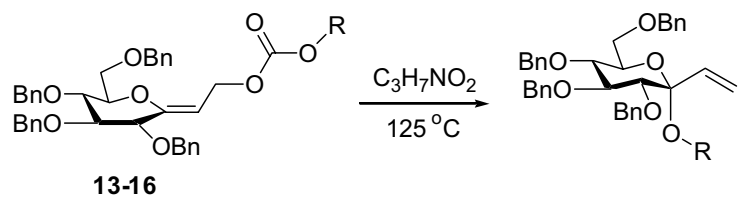

\begin{tabular}{llll}
\hline $\begin{array}{l}\text { Entry } \\
\text { (substrate) }\end{array}$ & $\begin{array}{l}\text { Intramolecular } \\
\text { acceptor }(\mathrm{O}-\mathrm{R})\end{array}$ & $\begin{array}{l}\text { Time } \\
(\mathrm{h})\end{array}$ & $\begin{array}{l}\text { Product } \\
\text { [yield (\%)] }\end{array}$ \\
\hline $\mathrm{i} \mathrm{(13)}$ & $\mathbf{9}(25)$ & 4 & $\mathbf{1 9}(20)$ \\
ii (14) & $\mathbf{2 0}(55)$
\end{tabular}

${ }^{a}$ All reaction were carried out in 1-nitropropane in the presence of molecular sieves ( 4 Å).

subjected to the reaction. The formation of the desired product was still observed in low yield. These unsatisfactory results indicate that the intramolecular glycosyl transfer is not favored in these reactions.

Ikegami et al. have used glycosyl carbonates for intramolecular glycosylation, ${ }^{21}$ and good yields with $\alpha$ - or $\beta$ stereochemical control were achieved by using different promoters. $^{22,23}$ In addition, Scheffler and Schmidt demonstrated with competition experiments that acidpromoted decarboxylative glycosylation (by using stoichiometric amount of TMSOTf or TBDMSOTf) follows an intermolecular course. ${ }^{24}$ In contrast to these other studies, there is a longer distance between the donor and acceptor used in our investigations. The carbonate is not directly attached to the anomeric center; instead, it is bridged by two additional carbons. The relative remoteness between the anomeric center and the nucleophile likely causes the low yields of the intramolecular glycosyl transfer even though the Lewis acid was able to increase the rate of decarboxylation. ${ }^{\dagger}$ The molecules used in our studies should adopt an extended conformation at high temperature. The longer lifetime of the decomposed carbonic acid monoester, if such an intermediate exists, is considered as another plausible cause. Whether there are other factors involved requires further investigation.

In conclusion, a decarboxylative glycosylation method that is carried out in $\mathrm{AlCl}_{3}$ in 1-nitropropane has been developed. The intermolecular reactions could be applied to a number of alcohol nucleophiles, and products with the $\alpha$-stereochemistry were formed exclusively and in good yield. We are further exploring this methodology and studies directed at understanding the theoretical basis of this reaction are in progress and will be published in a due course.

\section{Experimental procedures}

\subsection{General methods}

All reactions were conducted under an argon atmosphere. THF and diethyl ether were distilled from sodium benzophenone ketyl, $\mathrm{CH}_{2} \mathrm{Cl}_{2}$ was distilled from calcium hydride and $\mathrm{CH}_{3} \mathrm{OH}$ was distilled from magnesium. Solutions of compounds in organic solvents were dried over $\mathrm{Na}_{2} \mathrm{SO}_{4}$ prior to rotary evaporation. DMF was $99.5 \%$ pure and anhydrous. Benzyl bromide was filtered through alumina prior to use. TLC plates $(250 \mu \mathrm{m}$ of layer thickness) were Kieselgel $60 \mathrm{~F}_{254}$. Carbohydrate

\footnotetext{
${ }^{\dagger}$ In a control experiment that had no Lewis acid, most of the reactant remained unchanged after the workup. Although the addition of Lewis acid (e.g., $\mathrm{AlCl}_{3}, \mathrm{BF}_{3} \cdot \mathrm{OEt}_{2}$ or TMSOTf) afforded the desired product in a similar yield, a significant amount of the rearranged side product was obtained due to hydration.
} 
compounds were visualized with TLC by the stain solution $p$-anisaldehyde/ $\mathrm{H}_{2} \mathrm{SO}_{4} / \mathrm{EtOH}$ (6:1:100), phosphomolybdic acid (PMA)/EtOH (1:20), and $\mathrm{H}_{2} \mathrm{SO}_{4} / \mathrm{EtOH} /$ $\mathrm{H}_{2} \mathrm{O}(1: 10: 10)$. Column chromatography was carried out with Silica Gel 60 (70-230 mesh); gradients of EtOAc/hexanes and gradients of $\mathrm{CH}_{3} \mathrm{OH} / \mathrm{CHCl}_{3}$ were used as eluents. Optical rotations were measured on a digital polarimeter with a cuvette of $10 \mathrm{~cm}$ length. ${ }^{1} \mathrm{H}$ NMR spectra were recorded at 400 or $500 \mathrm{MHz}$ with $\mathrm{CHCl}_{3}\left(\delta_{\mathrm{H}} 7.24\right)$ or $\mathrm{CD}_{3} \mathrm{OD}\left[\delta_{\mathrm{H}} 3.30\right.$ (central line of a quintet)] as the internal standard; ${ }^{13} \mathrm{C}$ NMR spectra were recorded at 100 or $125 \mathrm{MHz}$ with $\mathrm{CDCl}_{3}\left[\delta_{\mathrm{C}} 77.0\right.$ (central line of a triplet)] or $\mathrm{CD}_{3} \mathrm{OD}\left[\delta_{\mathrm{C}} 49.0\right.$ (central line of a septet)] as the internal standard. Mass spectra were recorded with electrospray (ESI) or fast atom bombardment (FAB) ionization.

\section{2. (Z)-3,7-Anhydro-4,5,6,8-tetra-O-benzyl-1-O-tert- butoxycarbonyl-2,3-dideoxy-D-galacto-oct-2-enitol (1)}

To a solution of $17(1.45 \mathrm{~g}, 2.55 \mathrm{mmol})$ in $60 \mathrm{~mL}$ dry DMF were added imidazole-1-carboxylic acid tert-butyl ester ( $856 \mathrm{mg}, 5.1 \mathrm{mmol}$ ) and $\mathrm{NaH}$ (255 mg, $6.37 \mathrm{mmol}$ ) at $\mathrm{rt}$, and the solution was stirred for $1 \mathrm{~h}$. Water $(10 \mathrm{~mL})$ was added to quench the reaction and then the mixture was evaporated under reduced pressure. The crude residue was extracted by $\mathrm{CH}_{2} \mathrm{Cl}_{2}$ and the combined organic phase was dried $\left(\mathrm{MgSO}_{4}\right)$, concentrated, and purified by silica gel chromatography with EtOAc/hexanes (1:6) to give $1(1.528 \mathrm{~g}, 90 \%)$ as a colorless syrup; $R_{\mathrm{f}} 0.23$ (1:6, EtOAc/hexanes): $[\alpha]_{\mathrm{D}}+46.3\left(c 1.8, \mathrm{CH}_{2} \mathrm{Cl}_{2}\right)$; ${ }^{1} \mathrm{H} \mathrm{NMR}\left(\mathrm{CDCl}_{3}, 500 \mathrm{MHz}\right): \delta 7.34-7.24$ (20H, m, ArH), $5.34\left(1 \mathrm{H}, \quad \mathrm{t}, \quad J_{1^{\prime}, 2^{\prime}}=7.5 \mathrm{~Hz}, \quad \mathrm{H} 1^{\prime}\right), 4.90 \quad(1 \mathrm{H}, \mathrm{d}$, $\left.J_{\mathrm{a}, \mathrm{b}}=11.5 \mathrm{~Hz}, \quad \mathrm{CH}_{2} \mathrm{Ph}\right), 4.77\left(1 \mathrm{H}, \quad \mathrm{dd}, \quad J_{2 \mathrm{a}, 1}=8.0\right.$, $\left.J_{2 \mathrm{a}, 2 \mathrm{~b}}=12.0 \mathrm{~Hz}, \mathrm{H} 2 \mathrm{a}^{\prime}\right), 4.73\left(1 \mathrm{H}, \mathrm{d}, J_{\mathrm{a}, \mathrm{b}}=11.5 \mathrm{~Hz}\right.$, $\left.\mathrm{CH}_{2} \mathrm{Ph}\right), 4.70-4.66\left(4 \mathrm{H}, \mathrm{m}, \mathrm{CH}_{2} \mathrm{Ph}\right), 4.64(1 \mathrm{H}, \mathrm{dd}$, $\left.J_{2 \mathrm{~b}, 1}=6.0, J_{2 \mathrm{~b}, 2 \mathrm{a}}=12.0 \mathrm{~Hz}, \mathrm{H} 2 \mathrm{~b}^{\prime}\right), 4.58\left(1 \mathrm{H}, \mathrm{d}, J_{\mathrm{a}, \mathrm{b}}=\right.$ $\left.11.5 \mathrm{~Hz}, \mathrm{CH}_{2} \mathrm{Ph}\right), 4.50\left(1 \mathrm{H}, \mathrm{d}, J_{\mathrm{a}, \mathrm{b}}=12.0 \mathrm{~Hz}, \mathrm{CH}_{2} \mathrm{Ph}\right)$, $4.44\left(1 \mathrm{H}, \mathrm{d}, J_{\mathrm{a}, \mathrm{b}}=12.0 \mathrm{~Hz}, \mathrm{CH}_{2} \mathrm{Ph}\right), 4.33(1 \mathrm{H}, \mathrm{d}$, $\left.J_{2,3}=8.5 \mathrm{~Hz}, \mathrm{H} 2\right), 4.06\left(1 \mathrm{H}, \quad \mathrm{dd}, \quad J_{4,3}=2.5 \mathrm{~Hz}\right.$, $\left.J_{4,5}=2.5 \mathrm{~Hz}, \mathrm{H} 4\right), 3.85\left(1 \mathrm{H}, \mathrm{td}, J_{5,4}=2.5 \mathrm{~Hz}, J_{5,6}=\right.$ $6.0 \mathrm{~Hz}, \mathrm{H} 5), 3.73-3.64(3 \mathrm{H}, \mathrm{m}, \mathrm{H} 3, \mathrm{H} 6 \times 2), 1.45(9 \mathrm{H}, \mathrm{s}$, $\left.\mathrm{H} 3{ }^{\prime} \times 9\right) ;{ }^{13} \mathrm{C} \mathrm{NMR}\left(\mathrm{CDCl}_{3}, 100 \mathrm{MHz}\right): \delta 153.5,138.4$, $138.3,137.9,128.4,128.3,128.2,127.9,127.8,127.7$, $127.6,127.5,127.4,105.0,81.7,78.2,76.5,74.3,74.2$, 73.6, 73.4, 72.8, 68.3, 61.0, 60.3, 27.7; FABMS: $m / z$ calcd for $\mathrm{C}_{41} \mathrm{H}_{46} \mathrm{O}_{8} \mathrm{Na}[\mathrm{M}+\mathrm{Na}]^{+}:$689.3090. Found: 689.3089 .

\section{3. (Z)-3,7-Anhydro-4,5,6,8-tetra- $O$-benzyl-1-O-tert- butoxycarbonyl-2,3-dideoxy-D-gluco-oct-2-enitol (2)}

The compound was prepared in the same manner as $\mathbf{1}$. $[\alpha]_{\mathrm{D}}+47.3 \quad\left(c \quad 2.4, \mathrm{CH}_{2} \mathrm{Cl}_{2}\right) ;{ }^{1} \mathrm{H} \quad \mathrm{NMR} \quad\left(\mathrm{CDCl}_{3}\right.$, $400 \mathrm{MHz}): \delta$ 7.33-7.12 (20H, m, ArH), $5.22(1 \mathrm{H}, \mathrm{t}$, $\left.J_{1^{\prime}, 2^{\prime}}=7.6 \mathrm{~Hz}, \mathrm{H} 1^{\prime}\right), 4.82\left(1 \mathrm{H}, \mathrm{dd}, J_{2 \mathrm{a}^{\prime}, 1^{\prime}}=7.6 \mathrm{~Hz}\right.$, $\left.J_{2 \mathrm{a}^{\prime}, 2 \mathrm{~b}^{\prime}}=12.4 \mathrm{~Hz}, \mathrm{H} 2 \mathrm{a}^{\prime}\right), 4.81\left(1 \mathrm{H}, \mathrm{d}, J_{\mathrm{a}, \mathrm{b}}=11.6 \mathrm{~Hz}\right.$,
$\left.\mathrm{CH}_{2} \mathrm{Ph}\right), 4.75\left(1 \mathrm{H}, \mathrm{d}, \mathrm{J}_{\mathrm{a}, \mathrm{b}}=11.2 \mathrm{~Hz}, \mathrm{CH}_{2} \mathrm{Ph}\right), 4.73$ $\left(1 \mathrm{H}, \quad \mathrm{d}, \quad J_{\mathrm{a}, \mathrm{b}}=12.0 \mathrm{~Hz}, \quad \mathrm{CH}_{2} \mathrm{Ph}\right), 4.68 \quad(1 \mathrm{H}, \mathrm{d}$, $\left.J_{\mathrm{a}, \mathrm{b}}=12.8 \mathrm{~Hz}, \mathrm{CH} \mathrm{H}_{2} \mathrm{Ph}\right), 4.69-4.64\left(1 \mathrm{H}, \mathrm{m}, \mathrm{H} 2 \mathrm{~b}^{\prime}\right), 4.65$ $\left(1 \mathrm{H}, \quad \mathrm{d}, \quad J_{\mathrm{a}, \mathrm{b}}=12.0 \mathrm{~Hz}, \quad \mathrm{CH}_{2} \mathrm{Ph}\right), \quad 4.61 \quad(1 \mathrm{H}, \quad \mathrm{d}$, $\left.J_{\mathrm{a}, \mathrm{b}}=11.6 \mathrm{~Hz}, \mathrm{CH}_{2} \mathrm{Ph}\right), 4.52\left(1 \mathrm{H}, \mathrm{d}, J_{\mathrm{a}, \mathrm{b}}=12.4 \mathrm{~Hz}\right.$, $\left.\mathrm{CH}_{2} \mathrm{Ph}\right), 4.50\left(1 \mathrm{H}, \mathrm{d}, \mathrm{J}_{\mathrm{a}, \mathrm{b}}=10.8 \mathrm{~Hz}, \mathrm{CH}_{2} \mathrm{Ph}\right), 3.93$ $\left(1 \mathrm{H}, \mathrm{d}, J_{2,3}=7.2 \mathrm{~Hz}, \mathrm{H} 2\right), 3.84-3.73(4 \mathrm{H}, \mathrm{m}, \mathrm{H} 3, \mathrm{H} 5$, H6a,b), $3.69\left(1 \mathrm{H}, \mathrm{dd}, J_{4,3}=6.8 \mathrm{~Hz}, J_{4,5}=7.2 \mathrm{~Hz}, \mathrm{H} 4\right)$, $1.47\left(9 \mathrm{H}, \mathrm{s}, \mathrm{H} 3{ }^{\prime} \times 9\right) ;{ }^{13} \mathrm{C} \mathrm{NMR}\left(\mathrm{CDCl}_{3}, 100 \mathrm{MHz}\right): \delta$ $153.7,152.8,138.3,138.2,138.1,137.8,128.6,128.5$, $128.4,128.1,128.0,127.9,127.8,104.4,84.7,82.1$, 78.7, 78.4, 77.4, 74.6, 74.5, 73.6, 72.9, 68.5, 61.0, 28.0, 27.9; FABMS: $m / z$ calcd for $\mathrm{C}_{41} \mathrm{H}_{46} \mathrm{O}_{8} \mathrm{Na}[\mathrm{M}+\mathrm{Na}]^{+}$: 689.3090. Found: 689.3101.

\subsection{Typical glycosylation procedure (synthesis of 3)}

To a stirred solution of $\mathbf{1}$ ( $57 \mathrm{mg}, 0.085 \mathrm{mmol}$ ) in dry 1nitropropane $(2 \mathrm{~mL})$ containing $4 \AA$ molecular sieves were added allyl alcohol $(17.4 \mu \mathrm{L}, 0.25 \mathrm{mmol})$ and $\mathrm{AlCl}_{3}$ (11.3 mg, $0.085 \mathrm{mmol}$ ) at $0{ }^{\circ} \mathrm{C}$ under an argon atmosphere. After $40 \mathrm{~min}$, the reaction mixture was concentrated under reduced pressure and the resulting crude residue was purified by silica gel chromatography with EtOAc/hexanes (1:10) to give 3 (42 mg, 86\% yield) as a colorless syrup.

\subsection{Allyl 4,5,6,8-tetra- $O$-benzyl-1,2-dideoxy- $\alpha$-D- galacto-oct-3-ulo-1-enopyranoside (3)}

Yield: $86 \%$ as a colorless syrup; $R_{\mathrm{f}} 0.25(1: 10$, EtOAc/ hexanes); ${ }^{1} \mathrm{H}$ NMR $\left(\mathrm{CDCl}_{3}, 400 \mathrm{MHz}\right): \delta$ 7.36-7.25 (20H, m, ArH), 5.98-5.87 (2H, m, H1', H2'), 4.52 $\left(1 \mathrm{H}, \mathrm{dd}, J_{2 \mathrm{a}^{\prime}, 1^{\prime}}=17.6 \mathrm{~Hz}, J_{2 \mathrm{a}^{\prime}, 2 \mathrm{~b}^{\prime}}=1.6 \mathrm{~Hz}, \mathrm{H} 2 \mathrm{a}^{\prime}\right), 5.25$ $\left(1 \mathrm{H}, \quad \mathrm{dd}, \quad J_{3 \mathrm{a}^{\prime \prime}, 2^{\prime \prime}}=11.6 \mathrm{~Hz}, \quad J_{3 \mathrm{a}^{\prime \prime}, 3 \mathrm{~b}^{\prime \prime}}=1.6 \mathrm{~Hz}, \quad \mathrm{H} 3 \mathrm{a}^{\prime \prime}\right)$, $5.22\left(1 \mathrm{H}, \mathrm{dd}, J_{2 \mathrm{~b}^{\prime}, 1^{\prime}}=17.6 \mathrm{~Hz}, J_{2 \mathrm{~b}^{\prime}, 2 \mathrm{a}^{\prime}}=1.6 \mathrm{~Hz}, \mathrm{H} 2 \mathrm{~b}^{\prime}\right)$, $5.08\left(1 \mathrm{H}, \mathrm{dd}, J_{3 \mathrm{~b}^{\prime \prime}, 2^{\prime \prime}}=10.4 \mathrm{~Hz}, \mathrm{H} 3 \mathrm{~b}^{\prime \prime}\right), 4.95(1 \mathrm{H}, \mathrm{d}$, $\left.J_{\mathrm{a}, \mathrm{b}}=11.6 \mathrm{~Hz}, \mathrm{CH}_{2} \mathrm{Ph}\right), 4.87\left(1 \mathrm{H}, \mathrm{d}, J_{\mathrm{a}, \mathrm{b}}=11.2 \mathrm{~Hz}\right.$, $\left.\mathrm{CH}_{2} \mathrm{Ph}\right), 4.75\left(1 \mathrm{H}, \mathrm{d}, \mathrm{J}_{\mathrm{a}, \mathrm{b}}=11.6 \mathrm{~Hz}, \mathrm{CH}_{2} \mathrm{Ph}\right), 4.72$ $\left(1 \mathrm{H}, \quad \mathrm{d}, \quad J_{\mathrm{a}, \mathrm{b}}=11.6 \mathrm{~Hz}, \quad \mathrm{CH}_{2} \mathrm{Ph}\right), 4.63 \quad(1 \mathrm{H}, \mathrm{d}$, $\left.J_{\mathrm{a}, \mathrm{b}}=11.2 \mathrm{~Hz}, \mathrm{CH}_{2} \mathrm{Ph}\right), 4.60\left(1 \mathrm{H}, \mathrm{d}, J_{\mathrm{a}, \mathrm{b}}=11.6 \mathrm{~Hz}\right.$, $\left.\mathrm{CH}_{2} \mathrm{Ph}\right), 4.49\left(1 \mathrm{H}, \mathrm{d}, \mathrm{J}_{\mathrm{a}, \mathrm{b}}=12.0 \mathrm{~Hz}, \mathrm{CH}_{2} \mathrm{Ph}\right), 4.45$ $\left(1 \mathrm{H}, \mathrm{d}, J_{\mathrm{a}, \mathrm{b}}=12.0 \mathrm{~Hz}, \mathrm{CH}_{2} \mathrm{Ph}\right), 3.99-3.83(5 \mathrm{H}, \mathrm{m}, \mathrm{H} 2$, H4, H5, H6a, H6b), 3.64-3.55 (2H, m, H1a", H1b"); ${ }^{13} \mathrm{C}$ NMR $\left(\mathrm{CDCl}_{3}, 100 \mathrm{MHz}\right): \delta 138.9,138.7,138.4$, $138.1,135.2,135.0,128.3,128.1,127.9,127.6,127.4$, $118.6,116.2,100.2,80.4,80.3,75.7,75.0,74.4,73.3$, 72.8, 70.4, 69.0, 63.0; FABMS: $m / z$ calcd for $\mathrm{C}_{39} \mathrm{H}_{43} \mathrm{O}_{6}[\mathrm{M}+\mathrm{H}]^{+}:$607.3060. Found: 607.3063.

\subsection{Isopropyl 4,5,6,8-tetra- $O$-benzyl-1,2-dideoxy- $\alpha$-D- galacto-oct-3-ulo-1-enopyranoside (4)}

Yield: $71 \%$ as a colorless syrup; $R_{\mathrm{f}} 0.31$ (1:6, EtOAc/hexanes); ${ }^{1} \mathrm{H} \mathrm{NMR}\left(\mathrm{CDCl}_{3}, 400 \mathrm{MHz}\right): \delta 7.35-7.23(20 \mathrm{H}, \mathrm{m}$, $\mathrm{ArH}), 6.03\left(1 \mathrm{H}, \mathrm{dd}, J_{1^{\prime}, 2 \mathrm{a}^{\prime}}=17.6 \mathrm{~Hz}, J_{1^{\prime}, 2 \mathrm{~b}^{\prime}}=10.8 \mathrm{~Hz}\right.$, 
$\left.\mathrm{H} 1^{\prime}\right), 5.53\left(1 \mathrm{H}, \mathrm{dd}, J_{2 \mathrm{a}^{\prime}, 1^{\prime}}=17.6 \mathrm{~Hz}, J_{2 \mathrm{a}^{\prime}, 2 \mathrm{~b}^{\prime}}=2.0 \mathrm{~Hz}\right.$, $\left.\mathrm{H} 2 \mathrm{a}^{\prime}\right), 5.19\left(1 \mathrm{H}, \mathrm{dd}, J_{2 \mathrm{~b}^{\prime}, 1^{\prime}}=10.8 \mathrm{~Hz}, J_{2 \mathrm{~b}^{\prime}, 2 \mathrm{a}^{\prime}}=2.0 \mathrm{~Hz}\right.$, $\left.\mathrm{H}_{2} \mathrm{~b}^{\prime}\right), 4.94\left(1 \mathrm{H}, \mathrm{d}, J_{\mathrm{a}, \mathrm{b}}=12.0 \mathrm{~Hz}, \mathrm{CH}_{2} \mathrm{Ph}\right), 4.91(1 \mathrm{H}, \mathrm{d}$, $\left.J_{\mathrm{a}, \mathrm{b}}=12.0 \mathrm{~Hz}, \mathrm{CH}_{2} \mathrm{Ph}\right), 4.75\left(1 \mathrm{H}, \mathrm{d}, J_{\mathrm{a}, \mathrm{b}}=12.0 \mathrm{~Hz}\right.$, $\left.\mathrm{CH}_{2} \mathrm{Ph}\right), 4.72\left(1 \mathrm{H}, \mathrm{d}, J_{\mathrm{a}, \mathrm{b}}=12.0 \mathrm{~Hz}, \mathrm{CH}_{2} \mathrm{Ph}\right), 4.62(1 \mathrm{H}$, $\left.\mathrm{d}, J_{\mathrm{a}, \mathrm{b}}=11.6 \mathrm{~Hz}, \mathrm{CH}_{2} \mathrm{Ph}\right), 4.60\left(1 \mathrm{H}, \mathrm{d}, J_{\mathrm{a}, \mathrm{b}}=11.6 \mathrm{~Hz}\right.$, $\left.\mathrm{CH}_{2} \mathrm{Ph}\right), 4.51\left(1 \mathrm{H}, \mathrm{d}, J_{\mathrm{a}, \mathrm{b}}=12.0 \mathrm{~Hz}, \mathrm{CH}_{2} \mathrm{Ph}\right), 4.46(1 \mathrm{H}$, $\left.\mathrm{d}, J_{\mathrm{a}, \mathrm{b}}=12.0 \mathrm{~Hz}, \mathrm{CH}_{2} \mathrm{Ph}\right), 4.09-4.03(3 \mathrm{H}, \mathrm{m}, \mathrm{H} 3, \mathrm{H} 4$, $\mathrm{H} 5), 3.95\left(1 \mathrm{H}, \mathrm{m}, \mathrm{H}^{\prime \prime}\right), 3.78\left(1 \mathrm{H}, \mathrm{d}, J_{2,3}=9.6 \mathrm{~Hz}, \mathrm{H} 2\right)$, $3.66\left(1 \mathrm{H}, \mathrm{dd}, J_{6 \mathrm{a}, 6 \mathrm{~b}}=9.2 \mathrm{~Hz}, J_{6 \mathrm{a}, 5}=7.6 \mathrm{~Hz}, \mathrm{H} 6 \mathrm{a}\right), 3.58$ $\left(1 \mathrm{H}, \mathrm{d}, J_{6 \mathrm{~b}, 6 \mathrm{a}}=9.2 \mathrm{~Hz}, J_{6 \mathrm{~b}, 5}=5.6 \mathrm{~Hz}, \mathrm{H} 6 \mathrm{~b}\right), 1.16(3 \mathrm{H}$, $\left.\mathrm{d}, J=2.4 \mathrm{~Hz}, \mathrm{CH}_{3}\right), 1.15\left(3 \mathrm{H}, \mathrm{d}, J=2.8 \mathrm{~Hz}, \mathrm{CH}_{3}\right) ;{ }^{13} \mathrm{C}$ NMR $\left(\mathrm{CDCl}_{3}, 100 \mathrm{MHz}\right): \delta 139.1,138.8,138.2,136.6$, $128.3,128.2,128.1,127.9,127.8,127.6,127.4,127.3$, 117.4, 100.2, 81.2, 80.4, 75.5, 74.8, 74.4, 73.4, 72.6, 70.3, 69, 65.3, 24.1, 23.6; FABMS: $m / z$ calcd for $\mathrm{C}_{39} \mathrm{H}_{45} \mathrm{O}_{6}$ $[\mathrm{M}+\mathrm{H}]^{+}:$609.3216. Found: 609.3219.

\subsection{4,5,6,8-Tetra- $O$-benzyl-1,2-dideoxy- $\alpha$-D-galacto-oct- 3-ulo-1-enopyranosyl-(3 $\rightarrow$ 6)-1,2:3,4-diisopropylidine- $\alpha$-D-galactopyranose (5)}

Yield: $80 \%$ as a colorless syrup; $R_{\mathrm{f}} 0.33(1: 3$, EtOAc/ hexanes); $[\alpha]_{\mathrm{D}}+8.1\left(c 2.5, \mathrm{CH}_{2} \mathrm{Cl}_{2}\right) ;{ }^{1} \mathrm{H} \mathrm{NMR}\left(\mathrm{CDCl}_{3}\right.$, $400 \mathrm{MHz}): \delta$ 7.35-7.23 (20H, m, ArH), $5.95(1 \mathrm{H}, \mathrm{dd}$, $\left.J_{1^{\prime}, 2 \mathrm{a}^{\prime}}=17.6 \mathrm{~Hz}, J_{1^{\prime}, 2 \mathrm{~b}^{\prime}}=11.2 \mathrm{~Hz}, \mathrm{H} 1^{\prime}\right), 5.52(1 \mathrm{H}, \mathrm{dd}$, $\left.J_{2 \mathrm{a}^{\prime}, 1^{\prime}}=17.6 \mathrm{~Hz}, J_{2 \mathrm{a}^{\prime}, 2 \mathrm{~b}^{\prime}}=2.0 \mathrm{~Hz}, \mathrm{H} 2 \mathrm{a}^{\prime}\right), 5.48(1 \mathrm{H}, \mathrm{d}$, $\left.J_{1^{\prime \prime}, 2^{\prime \prime}}=4.8 \mathrm{~Hz}, \mathrm{H} 1^{\prime \prime}\right), 5.23\left(1 \mathrm{H}, \mathrm{dd}, J_{2 \mathrm{~b}^{\prime}, 1^{\prime}}=11.2 \mathrm{~Hz}\right.$, $\left.J_{2 \mathrm{~b}^{\prime}, 2 \mathrm{a}^{\prime}}=2.0 \mathrm{~Hz}, \mathrm{H} 2 \mathrm{~b}^{\prime}\right), 4.92\left(1 \mathrm{H}, \mathrm{d}, J_{\mathrm{a}, \mathrm{b}}=11.6 \mathrm{~Hz}\right.$, $\left.\mathrm{CH}_{2} \mathrm{Ph}\right), 4.87\left(1 \mathrm{H}, \mathrm{d}, J_{\mathrm{a}, \mathrm{b}}=11.2 \mathrm{~Hz}, \mathrm{CH}_{2} \mathrm{Ph}\right), 4.71(2 \mathrm{H}$, s, $\left.\mathrm{CH}_{2} \mathrm{Ph}\right), 4.61\left(1 \mathrm{H}, \mathrm{d}, J_{\mathrm{a}, \mathrm{b}}=11.6 \mathrm{~Hz}, \mathrm{CH}_{2} \mathrm{Ph}\right), 4.60$ $\left(1 \mathrm{H}, \quad \mathrm{d}, \quad J_{\mathrm{a}, \mathrm{b}}=11.6 \mathrm{~Hz}, \quad \mathrm{CH}_{2} \mathrm{Ph}\right), \quad 4.55 \quad(1 \mathrm{H}, \quad \mathrm{dd}$, $\left.J_{3^{\prime \prime}, 2^{\prime \prime}}=8.0 \mathrm{~Hz}, \quad J_{3^{\prime \prime}, 4^{\prime \prime}}=2.4 \mathrm{~Hz}, \quad \mathrm{H} 3^{\prime \prime}\right), 4.51(1 \mathrm{H}, \mathrm{d}$, $\left.J_{\mathrm{a}, \mathrm{b}}=11.6 \mathrm{~Hz}, \mathrm{CH}_{2} \mathrm{Ph}\right), 4.45\left(1 \mathrm{H}, \mathrm{d}, J_{\mathrm{a}, \mathrm{b}}=12.0 \mathrm{~Hz}\right.$, $\left.\mathrm{CH}_{2} \mathrm{Ph}\right), 4.27-4.23\left(2 \mathrm{H}, \mathrm{m}, \mathrm{H} 2^{\prime \prime}, \mathrm{H} 4^{\prime \prime}\right), 4.16(1 \mathrm{H}, \mathrm{m}$, H5), $4.10\left(1 \mathrm{H}, \mathrm{dd}, J_{3,2}=10.0 \mathrm{~Hz}, J_{3,4}=3.2 \mathrm{~Hz}, \mathrm{H} 3\right)$, $4.02\left(1 \mathrm{H}, \mathrm{dd}, J_{4,3}=3.2 \mathrm{~Hz}, J_{4,5}=1.2 \mathrm{~Hz}, \mathrm{H} 4\right), 3.98$ $\left(1 \mathrm{H}, \mathrm{m}, \mathrm{H} 5^{\prime \prime}\right), 3.84\left(1 \mathrm{H}, \mathrm{d}, J_{2,3}=10 \mathrm{~Hz}, \mathrm{H} 2\right), 3.68(1 \mathrm{H}$, $\left.\mathrm{dd}, J_{6 \mathrm{a}, 6 \mathrm{~b}}=9.2 \mathrm{~Hz}, \quad J_{6 \mathrm{a}, 5}=8.0 \mathrm{~Hz}, \mathrm{H} 6 \mathrm{a}\right), 3.61(1 \mathrm{H}$, dd, $\left.J_{6 \mathrm{a}^{\prime \prime}, 6 \mathrm{~b}^{\prime \prime}}=10.8, J_{6 \mathrm{a}^{\prime \prime}, 5^{\prime \prime}}=5.2 \mathrm{~Hz}, \mathrm{H} 6 \mathrm{a}^{\prime \prime}\right), 3.56(1 \mathrm{H}, \mathrm{dd}$, $\left.J_{6 \mathrm{~b}, 6 \mathrm{a}}=9.2 \mathrm{~Hz}, J_{6 \mathrm{~b}, 5}=5.6 \mathrm{~Hz}, \mathrm{H} 6 \mathrm{~b}\right), 3.50(1 \mathrm{H}, \mathrm{dd}$, $\left.J_{6 \mathrm{~b}^{\prime \prime}, 6 \mathrm{a}^{\prime \prime}}=10.8 \mathrm{~Hz}, J_{6 \mathrm{~b}^{\prime \prime}, 5^{\prime \prime}}=6.8 \mathrm{~Hz}, \mathrm{H} 6 \mathrm{~b}^{\prime \prime}\right), 1.49(3 \mathrm{H}, \mathrm{s}$, $\left.\mathrm{CH}_{3}\right), 1.38\left(3 \mathrm{H}, \mathrm{s}, \mathrm{CH}_{3}\right), 1.30\left(6 \mathrm{H}, \mathrm{s}, 2 \mathrm{CH}_{3}\right) ;{ }^{13} \mathrm{C} \mathrm{NMR}$ $\left(\mathrm{CDCl}_{3}, 100 \mathrm{MHz}\right): \delta 139.1,138.8,138.7,138.3,135.3$, $128.3,128.2,128.1,128,127.9,127.7,127.5,127.4$, 127.3, 118.7, 109.1, 108.4, 99.9, 96.2, 80.5, 80.2, 75.3, 75, $74.5,73.1,72.6,71.3,70.7,69.7,68.6,67.3,61.3,26.1$, 25.9; FABMS: $m / z$ calcd for $\mathrm{C}_{48} \mathrm{H}_{57} \mathrm{O}_{11}[\mathrm{M}+\mathrm{H}]^{+}$: 809.3901. Found: 809.3906.

\subsection{Methyl 4,5,6,8-tetra- $O$-benzyl-1,2-dideoxy- $\alpha$-D- galacto-oct-3-ulo-1-enopyranosyl-(3 $\rightarrow 6)-2,3,4-$ tri- $O$ - methyl- $\alpha$-D-glucopyranoside (6)}

Yield: $70 \%$ as a colorless syrup; $R_{\mathrm{f}} 0.26(1: 2.5$, EtOAc/ hexanes); $[\alpha]_{\mathrm{D}}+54.5\left(c 1.8, \mathrm{CH}_{2} \mathrm{Cl}_{2}\right) ;{ }^{1} \mathrm{H} \mathrm{NMR}\left(\mathrm{CDCl}_{3}\right.$,
$400 \mathrm{MHz}): \delta$ 7.35-7.24 (20H, m, ArH), $5.92(1 \mathrm{H}, \mathrm{dd}$, $\left.J_{1^{\prime}, 2 \mathrm{a}^{\prime}}=17.6 \mathrm{~Hz}, J_{1^{\prime}, 2 \mathrm{~b}^{\prime}}=10.8 \mathrm{~Hz}, \mathrm{H} 1^{\prime}\right), 5.52(1 \mathrm{H}, \mathrm{dd}$, $\left.J_{2 \mathrm{a}^{\prime}, 1^{\prime}}=17.6 \mathrm{~Hz}, J_{2 \mathrm{a}^{\prime}, 2 \mathrm{~b}^{\prime}}=2.0 \mathrm{~Hz}, \mathrm{H} 2 \mathrm{a}^{\prime}\right), 5.26(1 \mathrm{H}, \mathrm{dd}$, $\left.J_{2 \mathrm{~b}^{\prime}, 1^{\prime}}=10.8 \mathrm{~Hz}, J_{2 \mathrm{~b}^{\prime}, 2 \mathrm{a}^{\prime}}=2.0 \mathrm{~Hz}, \mathrm{H} 2 \mathrm{~b}^{\prime}\right), 4.96(1 \mathrm{H}, \mathrm{d}$, $\left.J_{\mathrm{a}, \mathrm{b}}=11.6 \mathrm{~Hz}, \mathrm{CH} H_{2} \mathrm{Ph}\right), 4.88\left(1 \mathrm{H}, \mathrm{d}, J_{\mathrm{a}, \mathrm{b}}=11.6 \mathrm{~Hz}\right.$, $\left.\mathrm{CH}_{2} \mathrm{Ph}\right), 4.69-4.60\left(5 \mathrm{H}, \mathrm{m}, \mathrm{H} 1^{\prime \prime}, \mathrm{CH}_{2} \mathrm{Ph}\right), 4.50(1 \mathrm{H}, \mathrm{d}$, $\left.J_{\mathrm{a}, \mathrm{b}}=11.6 \mathrm{~Hz}, \mathrm{CH}_{2} \mathrm{Ph}\right), 4.45\left(1 \mathrm{H}, \mathrm{d}, J_{\mathrm{a}, \mathrm{b}}=12.4 \mathrm{~Hz}\right.$, $\left.\mathrm{CH}_{2} \mathrm{Ph}\right), 4.07(1 \mathrm{H}, \mathrm{m}, \mathrm{H} 5), 4.03\left(1 \mathrm{H}, \mathrm{dd}, J_{3,2}=9.6 \mathrm{~Hz}\right.$, $\left.J_{3,4}=2.8 \mathrm{~Hz}, \mathrm{H} 3\right), 3.96(1 \mathrm{H}, \mathrm{m}, \mathrm{H} 4), 3.84(1 \mathrm{H}, \mathrm{d}$, $\left.J_{2,3}=10 \mathrm{~Hz}, \mathrm{H} 2\right), 3.69-3.56\left(4 \mathrm{H}, \mathrm{m}, \mathrm{H} 2^{\prime \prime}, \mathrm{H} 3^{\prime \prime}, \mathrm{H} 4^{\prime \prime}\right.$, $\left.\mathrm{H}^{\prime \prime}\right), 3.58\left(3 \mathrm{H}, \mathrm{s}, \mathrm{OCH}_{3}\right), 3.48-3.44(4 \mathrm{H}, \mathrm{m}, \mathrm{H} 6$, $\left.\mathrm{OCH}_{3}\right), \quad 3.42\left(3 \mathrm{H}, \quad \mathrm{s}, \quad \mathrm{OCH}_{3}\right), \quad 3.35 \quad(1 \mathrm{H}, \quad \mathrm{dd}$, $\left.J_{6 \mathrm{a}, 6 \mathrm{~b}}=10.8 \mathrm{~Hz}, \quad J_{6 \mathrm{a}, 5}=7.6 \mathrm{~Hz}, \quad \mathrm{H} 6\right), \quad 3.32(3 \mathrm{H}, \quad \mathrm{s}$, $\left.\mathrm{OCH}_{3}\right), 3.10\left(1 \mathrm{H}, \mathrm{dd}, J_{6 \mathrm{a}^{\prime}, 6 \mathrm{~b}^{\prime}}=9.6 \mathrm{~Hz}, J_{6 \mathrm{a}^{\prime \prime}, 5^{\prime \prime}}=3.6 \mathrm{~Hz}\right.$, H6a $\left.{ }^{\prime \prime}\right), 2.90\left(1 \mathrm{H}, \mathrm{dd}, J_{6 \mathrm{~b}^{\prime \prime}, 6 \mathrm{a}^{\prime \prime}}=10.0 \mathrm{~Hz}, J_{6 \mathrm{~b}^{\prime \prime}, 5^{\prime \prime}}=8.8 \mathrm{~Hz}\right.$, $\left.\mathrm{H}_{6}{ }^{\prime \prime}\right) ;{ }^{13} \mathrm{C}$ NMR $\left(\mathrm{CD}_{2} \mathrm{Cl}_{2}, 100 \mathrm{MHz}\right): \delta 139,138.7$, $138.6,138.3,135.2,128.3,128.1,128,127.9,127.5$, $127.4,127.3,118.4,99.8,96.8,83.5,81.8,80.5,79.7$, $75.2,75.1,74.4,73.1,72.5,70.1,69.7,69.0,61.4,60.7$, 60.2, 58.8, 54.7; FABMS: $m / z$ calcd for $\mathrm{C}_{46} \mathrm{H}_{57} \mathrm{O}_{11}$ $[\mathrm{M}+\mathrm{H}]^{+}:$785.3901. Found: 785.3898 .

3.9. $O$-(4,5,6,8-Tetra- $O$-benzyl-1,2-dideoxy- $\alpha$-D-galactooct-3-ulo-1-enopyranosyl)- $N$-tert-butoxycarbonyl-Lserine methyl ester (7)

Yield: $75 \%$ as a colorless syrup; $R_{\mathrm{f}} 0.27(1: 4$, EtOAc/ hexanes); $[\alpha]_{\mathrm{D}}+18$ (c 2.2, $\left.\mathrm{CH}_{2} \mathrm{Cl}_{2}\right) ;{ }^{1} \mathrm{H} \mathrm{NMR}\left(\mathrm{CDCl}_{3}\right.$, $400 \mathrm{MHz}): \delta 7.33-7.21(20 \mathrm{H}, \mathrm{m}, \mathrm{ArH}), 5.81(1 \mathrm{H}, \mathrm{dd}$, $\left.J_{1^{\prime}, 2 \mathrm{a}^{\prime}}=10.8 \mathrm{~Hz}, J_{1^{\prime}, 2 \mathrm{~b}^{\prime}}=17.6 \mathrm{~Hz}, \mathrm{H} 1^{\prime}\right), 5.49(1 \mathrm{H}, \mathrm{dd}$, $\left.J_{2 \mathrm{a}^{\prime}, 1^{\prime}}=17.2 \mathrm{~Hz}, J_{2 \mathrm{a}^{\prime}, 2 \mathrm{~b}^{\prime \prime}}=1.2 \mathrm{~Hz}, \mathrm{H} 2 \mathrm{a}^{\prime}\right), 5.37(1 \mathrm{H}, \mathrm{d}$, $J=8.4 \mathrm{~Hz}, \quad \mathrm{NH}), \quad 5.28 \quad\left(1 \mathrm{H}, \quad \mathrm{dd}, \quad J_{2 \mathrm{~b}^{\prime}, 2 \mathrm{a}^{\prime}}=1.6 \mathrm{~Hz}\right.$, $\left.J_{2 \mathrm{~b}^{\prime}, 1^{\prime}}=11.2 \mathrm{~Hz}, \mathrm{H} 2 \mathrm{~b}^{\prime}\right), 4.92\left(1 \mathrm{H}, \mathrm{d}, J_{\mathrm{a}, \mathrm{b}}=11.6 \mathrm{~Hz}\right.$, $\left.\mathrm{CH}_{2} \mathrm{Ph}\right), 4.86\left(1 \mathrm{H}, \mathrm{d}, J_{\mathrm{a}, \mathrm{b}}=11.6 \mathrm{~Hz}, \mathrm{CH}_{2} \mathrm{Ph}\right), 4.71$ $\left(2 \mathrm{H}, \mathrm{s}, \mathrm{CH}_{2} \mathrm{Ph}\right), 4.58\left(1 \mathrm{H}, \mathrm{d}, J_{\mathrm{a}, \mathrm{b}}=12 \mathrm{~Hz}, \mathrm{CH}_{2} \mathrm{Ph}\right)$, $4.53\left(1 \mathrm{H}, \mathrm{d}, J_{\mathrm{a}, \mathrm{b}}=11.2 \mathrm{CH}_{2} \mathrm{Ph}\right), 4.50-4.42(3 \mathrm{H}, \mathrm{m}$, $\left.\mathrm{H} 2^{\prime \prime}, \mathrm{CH}_{2} \mathrm{Ph} \times 2\right), 3.98-3.96(2 \mathrm{H}, \mathrm{m}, \mathrm{H} 2, \mathrm{H} 3), 3.86-$ $3.75(3 \mathrm{H}, \mathrm{m}, \mathrm{H} 4, \mathrm{H} 5, \mathrm{H} 6), 3.59\left(3 \mathrm{H}, \mathrm{s}, \mathrm{COOCH}_{3}\right)$, 3.57-3.51 (3H, m, H6, H1a", b"), $1.42\left(9 \mathrm{H}, \mathrm{s}, 3 \mathrm{CH}_{3}\right)$; ${ }^{13} \mathrm{C} \mathrm{NMR}\left(\mathrm{CDCl}_{3}, 100 \mathrm{MHz}\right): \delta 171.0,138.9,138.8$, $138.6,138,134.5,128.3,128.1,127.9,127.7,127.6$, $127.5,127.4,127.2,119.3,99.9,80.5,79.7,75.2,74.7$, $74.5,73.4,72.6,70.5,68.8,62.4,53.9,52.2,28.3$; FABMS: $m / z$ calcd for $\mathrm{C}_{45} \mathrm{H}_{53} \mathrm{O}_{10} \mathrm{Na} \quad[\mathrm{M}+\mathrm{Na}]^{+}$: 790.3576. Found: 790.3574.

\subsection{0. $O$-(4,5,6,8-Tetra- $O$-benzyl-1,2-dideoxy- $\alpha$-D-} galacto-oct-3-ulo-1-enopyranosyl)- $N$ - $\alpha$-(9-fluorenylmethyloxycarbonyl)-L-threonine methyl ester (8)

Yield: $60 \%$ as a white solid; $R_{\mathrm{f}} 0.2(1: 5$, EtOAc/hexanes); ${ }^{1} \mathrm{H}$ NMR $\left(\mathrm{CDCl}_{3}, 400 \mathrm{MHz}\right): \delta 7.74(2 \mathrm{H}, \mathrm{d}, J=7.6 \mathrm{~Hz}$, ArH), $7.60(2 \mathrm{H}, \mathrm{dd}, J=2.8,7.2 \mathrm{~Hz}, \mathrm{ArH}), 7.37-7.21$ $(24 \mathrm{H}, \quad \mathrm{m}, \quad \mathrm{ArH}), \quad 5.87\left(1 \mathrm{H}, \quad \mathrm{dd}, J_{1^{\prime}, 2 \mathrm{a}^{\prime}}=11.2 \mathrm{~Hz}\right.$, $\left.J_{1^{\prime}, 2 \mathrm{a}^{\prime}}=17.6 \mathrm{~Hz}, \mathrm{H}^{\prime}\right), 5.71(1 \mathrm{H}, \mathrm{d}, J=9.2 \mathrm{~Hz}, \mathrm{NH})$, $5.59\left(1 \mathrm{H}, \mathrm{dd}, J_{2 \mathrm{a}^{\prime}, 2 \mathrm{~b}^{\prime}}=1.6 \mathrm{~Hz}, J_{2 \mathrm{a}^{\prime}, 1^{\prime}}=17.6 \mathrm{~Hz}, \mathrm{H} 2 \mathrm{a}^{\prime}\right)$, 
$5.31\left(1 \mathrm{H}, \mathrm{dd}, J_{2 \mathrm{~b}^{\prime}, 2 \mathrm{a}^{\prime}}=1.6 \mathrm{~Hz}, J_{2 \mathrm{~b}^{\prime}, 1^{\prime}}=10.8 \mathrm{~Hz}, \mathrm{H} 2 \mathrm{~b}^{\prime}\right)$, $4.95\left(1 \mathrm{H}, \mathrm{d}, J_{\mathrm{a}, \mathrm{b}}=11.6 \mathrm{~Hz}, \mathrm{CH}_{2} \mathrm{Ph}\right), 4.87(1 \mathrm{H}, \mathrm{d}$, $\left.J_{\mathrm{a}, \mathrm{b}}=10.8 \mathrm{~Hz}, \mathrm{C} \mathrm{H}_{2} \mathrm{Ph}\right), 4.75\left(2 \mathrm{H}, \mathrm{s}, \mathrm{CH}_{2} \mathrm{Ph}\right), 4.61$ $\left(1 \mathrm{H}, \quad \mathrm{d}, \quad J_{\mathrm{a}, \mathrm{b}}=11.6 \mathrm{~Hz}, \quad \mathrm{CH}_{2} \mathrm{Ph}\right), \quad 4.54(1 \mathrm{H}, \quad \mathrm{d}$, $\left.J_{\mathrm{a}, \mathrm{b}}=11.2 \mathrm{~Hz}, \quad \mathrm{CH}_{2} \mathrm{Ph}\right), \quad 4.50-4.33 \quad\left(4 \mathrm{H}, \quad \mathrm{m}, \quad \mathrm{H} 2^{\prime \prime}\right.$, $\left.\mathrm{H}^{4} \mathrm{a}^{\prime \prime}, 4 \mathrm{~b}^{\prime \prime}, \mathrm{CH} \mathrm{H}_{2} \mathrm{Ph}\right), 4.26-4.23\left(2 \mathrm{H}, \mathrm{m}, \mathrm{H} 3^{\prime \prime}, \mathrm{H} 5^{\prime \prime}\right), 4.05-$ $3.98(3 \mathrm{H}, \mathrm{m}, \mathrm{H} 3, \mathrm{H} 4, \mathrm{H} 5), 3.76\left(1 \mathrm{H}, \mathrm{d}, J_{2,3}=9.6 \mathrm{~Hz}\right.$, $\mathrm{H} 2), 3.64\left(1 \mathrm{H}, \quad \mathrm{dd}, J_{6 \mathrm{a}, 5}=7.2 \mathrm{~Hz}, J_{6 \mathrm{a}, 6 \mathrm{~b}}=9.2 \mathrm{~Hz}\right.$, $\mathrm{H} 6 \mathrm{a}), 3.54\left(1 \mathrm{H}, \mathrm{dd}, J_{6 \mathrm{~b}, 5}=6 \mathrm{~Hz}, J_{6 \mathrm{~b}, 6 \mathrm{a}}=9.2 \mathrm{~Hz}, \mathrm{H} 6 \mathrm{~b}\right)$, $3.45\left(3 \mathrm{H}, \mathrm{s}, \mathrm{COOCH} H_{3}\right), 1.61\left(3 \mathrm{H}, \mathrm{d}, J=6.4 \mathrm{~Hz} \mathrm{CH} \mathrm{CH}_{3}\right)$; ${ }^{13} \mathrm{C} \mathrm{NMR}\left(\mathrm{CDCl}_{3}, 100 \mathrm{MHz}\right): \delta 170.8,156.7,144.0$, $143.8,141.2,138.9,138.5,138.0,134.4,128.4,128.3$, $128.1,127.8,127.7,127.6,127.5,127.4,127.1,127.0$, $125.1,119.9,119.0,100.3,81.0,79.6,75.7,74.7,74.4$, 73.5, 72.5, 70.4, 69.8, 68.8, 67.1, 59.5, 52.1, 47.2, 18.7; FABMS: $m / z$ calcd for $\mathrm{C}_{56} \mathrm{H}_{58} \mathrm{NO}_{10} \quad[\mathrm{M}+\mathrm{H}]^{+}$: 904.4061. Found: 904.4056.

\subsection{1. $n$-Butyl 4,5,6,8-tetra- $O$-benzyl-1,2-dideoxy- $\alpha$-D- gluco-oct-3-ulo-1-enopyranoside (9)}

Yield: $80 \%$ as a colorless syrup; $R_{\mathrm{f}} 0.24(1: 15$, EtOAc/ hexanes); ${ }^{1} \mathrm{H}$ NMR $\left(\mathrm{CD}_{2} \mathrm{Cl}_{2}, 400 \mathrm{MHz}\right): \delta$ 7.46-7.33 $(20 \mathrm{H}, \quad \mathrm{m}, \quad \mathrm{ArH}), \quad 5.99\left(1 \mathrm{H}, \quad \mathrm{dd}, \quad J_{1^{\prime}, 2 \mathrm{a}^{\prime}}=10.8 \mathrm{~Hz}\right.$, $\left.J_{1^{\prime}, 2 \mathrm{~b}^{\prime}}=17.6 \mathrm{~Hz}, \mathrm{H} 1^{\prime}\right), 5.54\left(1 \mathrm{H}, \mathrm{dd}, J_{2 \mathrm{~b}^{\prime}, 2 \mathrm{a}^{\prime}}=2.0 \mathrm{~Hz}\right.$, $\left.J_{2 \mathrm{~b}^{\prime}, 1^{\prime}}=17.6 \mathrm{~Hz}, \mathrm{H} 2 \mathrm{~b}^{\prime}\right), 5.35\left(1 \mathrm{H}, \mathrm{dd}, J_{2 \mathrm{a}^{\prime}, 2 \mathrm{~b}^{\prime}}=2.0 \mathrm{~Hz}\right.$, $\left.J_{2 \mathrm{a}^{\prime}, 1^{\prime}}=10.8 \mathrm{~Hz}, \mathrm{H} 2 \mathrm{a}^{\prime}\right), 4.98\left(1 \mathrm{H}, \mathrm{d}, J_{\mathrm{a}, \mathrm{b}}=11.2 \mathrm{~Hz}\right.$, $\left.\mathrm{CH}_{2} \mathrm{Ph}\right), 4.89\left(1 \mathrm{H}, \mathrm{d}, J_{\mathrm{a}, \mathrm{b}}=10.8 \mathrm{~Hz}, \mathrm{CH}_{2} \mathrm{Ph}\right), 4.84$ $\left(1 \mathrm{H}, \quad \mathrm{d}, \quad J_{\mathrm{a}, \mathrm{b}}=12.0 \mathrm{~Hz}, \quad \mathrm{CH}_{2} \mathrm{Ph}\right), \quad 4.79(1 \mathrm{H}, \quad \mathrm{d}$, $\left.J_{\mathrm{a}, \mathrm{b}}=11.6 \mathrm{~Hz}, \mathrm{CH} H_{2} \mathrm{Ph}\right), 4.62\left(1 \mathrm{H}, \mathrm{d}, J_{\mathrm{a}, \mathrm{b}}=10.4 \mathrm{~Hz}\right.$, $\left.\mathrm{CH}_{2} \mathrm{Ph}\right), 4.61\left(1 \mathrm{H}, \mathrm{d}, J_{\mathrm{a}, \mathrm{b}}=11.2 \mathrm{~Hz}, \mathrm{CH}_{2} \mathrm{Ph}\right), 4.60$ $\left(1 \mathrm{H}, \quad \mathrm{d}, \quad J_{\mathrm{a}, \mathrm{b}}=12.0 \mathrm{~Hz}, \quad \mathrm{CH}_{2} \mathrm{Ph}\right), \quad 5.54(1 \mathrm{H}, \quad \mathrm{d}$, $\left.J_{\mathrm{a}, \mathrm{b}}=12.0 \mathrm{~Hz}, \mathrm{CH} \mathrm{H}_{2} \mathrm{Ph}\right), 4.13-4.08(2 \mathrm{H}, \mathrm{m}, \mathrm{H} 3, \mathrm{H} 5)$, $3.94\left(1 \mathrm{H}, \quad \mathrm{dd}, \quad J_{4,3}=6.0 \mathrm{~Hz}, \mathrm{H} 4\right), 3.80(1 \mathrm{H}, \quad \mathrm{d}$, $\left.J_{2,3}=9.6 \mathrm{~Hz}, \quad \mathrm{H} 2\right), \quad 3.71\left(1 \mathrm{H}, \quad \mathrm{dd}, \quad J_{6 \mathrm{a}, 5}=7.2 \mathrm{~Hz}\right.$, $\left.J_{6 \mathrm{a}, 6 \mathrm{~b}}=9.6 \mathrm{~Hz}, \mathrm{H} 6 \mathrm{a}\right), 3.65\left(1 \mathrm{H}, \mathrm{dd}, J_{6 \mathrm{~b}, 5}=6.0 \mathrm{~Hz}\right.$, $\left.J_{6 \mathrm{~b}, 6 \mathrm{a}}=9.2 \mathrm{~Hz}, \mathrm{H} 6 \mathrm{~b}\right), 3.44-3.31\left(2 \mathrm{H}, \mathrm{m}, \mathrm{H}_{1} \mathrm{a}^{\prime \prime}, \mathrm{H}^{\prime} \mathrm{b}^{\prime \prime}\right.$ ), 1.62-1.56 (2H, m, H2a", H2b"), 1.43-1.34 (2H, m, $\left.\mathrm{H} 3 \mathrm{a}^{\prime \prime}, \mathrm{H} 3 \mathrm{~b}^{\prime \prime}\right), 0.96\left(3 \mathrm{H}, \mathrm{t}, \quad J=11.6 \mathrm{~Hz}, \mathrm{CH}_{3}\right) ;{ }^{13} \mathrm{C}$ NMR $\left(\mathrm{CD}_{2} \mathrm{Cl}_{2}, 100 \mathrm{MHz}\right): \delta \quad 138.9,138.8,138.6$, $138.3,135.6,128.2,128.1,128,127.5,127.4,127.3$, $117.9,99.5,80.7,80.1,75.6,75.2,74.7,73.2,72.5,70.1$, 69, 61.2, 31.7, 29.6, 19.5, 13.7, 10.7; FABMS: $m / z$ calcd for $\mathrm{C}_{40} \mathrm{H}_{46} \mathrm{NaO}_{6}[\mathrm{M}+\mathrm{Na}]^{+}$: 645.3192. Found: 645.3195.

3.12. 4,5,6,8-Tetra- $O$-benzyl-1,2-dideoxy- $\alpha$-D-gluco-oct-3ulo-1-enopyranosyl-(3 $\rightarrow 6)$-1,2:3,4-diisopropylidine- $\alpha$-Dgalactopyranose (10)

Yield: $74 \%$ as a colorless syrup; $[\alpha]_{\mathrm{D}}+5.0$ (c 2.2, $\left.\mathrm{CH}_{2} \mathrm{Cl}_{2}\right) ;{ }^{1} \mathrm{H}$ NMR $\left(\mathrm{CDCl}_{3}, 400 \mathrm{MHz}\right): \delta$ 7.34-7.2 $(20 \mathrm{H}, \quad \mathrm{m}, \quad \mathrm{ArH}), \quad 5.99\left(1 \mathrm{H}, \quad \mathrm{dd}, \quad J_{1^{\prime}, 2 \mathrm{a}^{\prime}}=17.2 \mathrm{~Hz}\right.$, $\left.J_{1^{\prime}, 2 \mathrm{~b}^{\prime}}=10.8 \mathrm{~Hz}, \mathrm{H} 1^{\prime}\right), 5.58\left(1 \mathrm{H}, \mathrm{dd}, J_{2 \mathrm{a}^{\prime}, 1^{\prime}}=17.2 \mathrm{~Hz}\right.$, $\left.J_{2 \mathrm{a}^{\prime}, 2 \mathrm{~b}^{\prime}}=2.0 \mathrm{~Hz}, \mathrm{H} 2 \mathrm{a}^{\prime}\right), 5.48\left(1 \mathrm{H}, \mathrm{d}, J_{1^{\prime \prime}, 2^{\prime \prime}}=4.8 \mathrm{~Hz}\right.$, $\left.\mathrm{H}^{\prime \prime}\right), 5.28\left(1 \mathrm{H}, \mathrm{dd}, J_{2 \mathrm{~b}^{\prime}, 1^{\prime}}=10.8 \mathrm{~Hz}, J_{2 \mathrm{~b}^{\prime}, 2 \mathrm{a}^{\prime}}=2.0 \mathrm{~Hz}\right.$, $\left.\mathrm{H} 2 \mathrm{~b}^{\prime}\right), 4.88-4.81\left(4 \mathrm{H}, \mathrm{m}, \mathrm{CH}_{2} \mathrm{Ph} \times 4\right), 4.66-4.53(5 \mathrm{H}$, $\left.\mathrm{m}, \quad \mathrm{H} 3^{\prime \prime}, \quad \mathrm{CH}_{2} \mathrm{Ph} \times 4\right), \quad 4.30\left(1 \mathrm{H}, \mathrm{dd}, \quad J_{2^{\prime \prime}, 3^{\prime \prime}}=8 \mathrm{~Hz}\right.$, $\left.J_{2^{\prime \prime}, 1^{\prime \prime}}=2 \mathrm{~Hz}, \quad \mathrm{H} 2^{\prime \prime}\right), \quad 4.26\left(1 \mathrm{H}, \quad \mathrm{dd}, \quad J_{4^{\prime \prime}, 3^{\prime \prime}}=5.2 \mathrm{~Hz}\right.$, $\left.J_{4^{\prime \prime}, 5^{\prime \prime}}=2.4 \mathrm{~Hz}, \quad \mathrm{H} 4^{\prime \prime}\right), \quad 4.10 \quad\left(1 \mathrm{H}, \quad \mathrm{dd}, \quad J_{3,2}=9.2 \mathrm{~Hz}\right.$, $\left.J_{3,4}=9.2 \mathrm{~Hz}, \mathrm{H} 3\right), 3.98\left(1 \mathrm{H}, \mathrm{m}, \mathrm{H} 5^{\prime \prime}\right), 3.92(1 \mathrm{H}, \mathrm{m}$, $\mathrm{H} 5), \quad 3.82\left(1 \mathrm{H}, \quad \mathrm{dd}, J_{6 \mathrm{a}, 6 \mathrm{~b}}=11.2 \mathrm{~Hz}, J_{6 \mathrm{a}, 5}=3.6 \mathrm{~Hz}\right.$, H6a), 3.73-3.64 (3H, m, H6b, H6a", H6b"), $3.5(1 \mathrm{H}$, $\left.\mathrm{dd}, J_{4,3}=10.0 \mathrm{~Hz}, J_{4,5}=5.6 \mathrm{~Hz}, \mathrm{H} 4\right), 3.35(1 \mathrm{H}, \mathrm{d}$, $\left.J_{2,3}=9.2 \mathrm{~Hz}, \mathrm{H} 2\right), 1.66\left(3 \mathrm{H}, \mathrm{s}, \mathrm{CH}_{3}\right), 1.40(3 \mathrm{H}, \mathrm{s}$, $\left.\mathrm{CH}_{3}\right), 1.31\left(3 \mathrm{H}, \mathrm{s}, \mathrm{CH}_{3}\right), 1.30\left(3 \mathrm{H}, \mathrm{s}, \mathrm{CH}_{3}\right) ;{ }^{13} \mathrm{C} \mathrm{NMR}$ $\left(\mathrm{CDCl}_{3}, 100 \mathrm{MHz}\right): \delta 138.8,138.6,138.4,135.6,128.3$, $128.2,128.1,127.9,127.8,127.7,127.6,127.5,127.4$, $118.8,109,108.5,99.8,96.2,84.6,82.7,78.4,75.4$, $74.5,73.3,71.5,71.1,70.7,70.6,68.8,67.5,67.1,61.2$, 26.1, 25.9, 24.9, 24.4; FABMS: $\mathrm{m} / \mathrm{z}$ calcd for $\mathrm{C}_{48} \mathrm{H}_{57} \mathrm{O}_{11}[\mathrm{M}+\mathrm{H}]^{+}:$809.3901. Found: 809.3908.

\subsection{Methyl 4,5,6,8-tetra- $O$-benzyl-1,2-dideoxy- $\alpha$-D- gluco-oct-3-ulo-1-enopyranosyl-(3 $\rightarrow 6)-2,3,4$-tri- $O$ - methyl- $\alpha$-D-glucopyranoside (11)}

Yield: $68 \%$ as a colorless syrup; $[\alpha]_{\mathrm{D}}+84.5$ (c 1.9, $\left.\mathrm{CH}_{2} \mathrm{Cl}_{2}\right) ;{ }^{1} \mathrm{H}$ NMR $\left(\mathrm{CD}_{2} \mathrm{Cl}_{2}, 400 \mathrm{MHz}\right): \delta 7.41-7.26$ $(20 \mathrm{H}, \quad \mathrm{m}, \quad \mathrm{ArH}), \quad 6.04 \quad\left(1 \mathrm{H}, \quad \mathrm{dd}, \quad J_{1^{\prime}, 2 \mathrm{a}^{\prime}}=11.2 \mathrm{~Hz}\right.$, $\left.J_{1^{\prime}, 2 \mathrm{~b}^{\prime}}=17.6 \mathrm{~Hz}, \mathrm{H} 1^{\prime}\right), 5.63\left(1 \mathrm{H}, \mathrm{dd}, J_{2 \mathrm{~b}^{\prime}, 2 \mathrm{a}^{\prime}}=1.6 \mathrm{~Hz}\right.$, $\left.J_{2 \mathrm{~b}^{\prime}, 1^{\prime}}=17.2 \mathrm{~Hz}, \mathrm{H} 2 \mathrm{~b}^{\prime}\right), 5.42\left(1 \mathrm{H}, \mathrm{dd}, J_{2 \mathrm{a}^{\prime}, 2 \mathrm{~b}^{\prime}}=2.0 \mathrm{~Hz}\right.$, $\left.J_{2 \mathrm{a}^{\prime}, 1^{\prime}}=10.8 \mathrm{~Hz}, \mathrm{H} 2 \mathrm{a}^{\prime}\right), 4.94\left(1 \mathrm{H}, \mathrm{d}, J_{\mathrm{a}, \mathrm{b}}=10.8 \mathrm{~Hz}\right.$, $\left.\mathrm{CH}_{2} \mathrm{Ph}\right), 4.91\left(1 \mathrm{H}, \mathrm{d}, J_{\mathrm{a}, \mathrm{b}}=11.6 \mathrm{~Hz}, \mathrm{CH}_{2} \mathrm{Ph}\right), 4.88$ $\left(1 \mathrm{H}, \quad \mathrm{d}, \quad J_{\mathrm{a}, \mathrm{b}}=10.8 \mathrm{~Hz}, \quad \mathrm{CH}_{2} \mathrm{Ph}\right), \quad 4.87(1 \mathrm{H}, \quad \mathrm{d}$, $\left.J_{\mathrm{a}, \mathrm{b}}=11.2 \mathrm{~Hz}, \mathrm{CH}_{2} \mathrm{Ph}\right), 4.80\left(1 \mathrm{H}, \mathrm{d}, J_{1^{\prime \prime}, 2^{\prime \prime}}=3.6 \mathrm{~Hz}\right.$, $\left.\mathrm{H}^{\prime \prime}\right), 4.67\left(1 \mathrm{H}, \mathrm{d}, J_{\mathrm{a}, \mathrm{b}}=10.0 \mathrm{~Hz}, \mathrm{CH}_{2} \mathrm{Ph}\right), 4.68(1 \mathrm{H}$, $\left.\mathrm{d}, J_{\mathrm{a}, \mathrm{b}}=12.0 \mathrm{~Hz}, \mathrm{CH}_{2} \mathrm{Ph}\right), 4.64\left(1 \mathrm{H}, \mathrm{d}, J_{\mathrm{a}, \mathrm{b}}=10.4 \mathrm{~Hz}\right.$, $\left.\mathrm{CH}_{2} \mathrm{Ph}\right), 4.61\left(1 \mathrm{H}, \mathrm{d}, J_{\mathrm{a}, \mathrm{b}}=13.2 \mathrm{~Hz}, \mathrm{CH}_{2} \mathrm{Ph}\right), 4.1(1 \mathrm{H}$, $\left.\mathrm{dd}, J_{3,2}=9.2 \mathrm{~Hz}, J_{3,4}=9.6 \mathrm{~Hz}, \mathrm{H} 3\right), 4.02(1 \mathrm{H}, \mathrm{ddd}$, $\left.J_{5^{\prime \prime}, 4^{\prime \prime}}=1.6 \mathrm{~Hz}, J_{5^{\prime \prime}, 6 \mathrm{a}^{\prime \prime}}=4.0 \mathrm{~Hz}, J_{5^{\prime \prime}, 6 \mathrm{~b}^{\prime \prime}}=10.0 \mathrm{~Hz}, \mathrm{H} 5^{\prime \prime}\right)$, 3.84-3.37 (8H, m, H3, H4, H5, H6A, H6B, H4", $\left.\mathrm{H} 6^{\prime \prime} \mathrm{A}, \mathrm{H} 6^{\prime \prime} \mathrm{B}\right), 3.60\left(3 \mathrm{H}, \quad \mathrm{s}, \mathrm{OCH}_{3}\right), 3.52(3 \mathrm{H}, \mathrm{s}$, $\left.\mathrm{OCH}_{3}\right), 3.47\left(3 \mathrm{H}, \mathrm{s}, \mathrm{OCH}_{3}\right), 3.40\left(3 \mathrm{H}, \mathrm{s}, \mathrm{OCH}_{3}\right), 3.20$ $\left(1 \mathrm{H}, \mathrm{dd}, J_{2^{\prime \prime}, 1^{\prime \prime}}=4.0 \mathrm{~Hz}, J_{2^{\prime \prime}, 3^{\prime \prime}}=10.4 \mathrm{~Hz}, \mathrm{H} 2^{\prime \prime}\right), 3.03$ $\left(1 \mathrm{H}, \quad \mathrm{dd}, J_{3^{\prime \prime}, 4^{\prime \prime}}=8.8 \mathrm{~Hz}, J_{3^{\prime \prime}, 2^{\prime \prime}}=10.0 \mathrm{~Hz}, \mathrm{H}^{\prime \prime}\right) ;{ }^{13} \mathrm{C}$ NMR $\left(\mathrm{CD}_{2} \mathrm{Cl}_{2}, 100 \mathrm{MHz}\right): \delta \quad 138.8,138.6,138.2$, $135.3,128.2,128.1,128,127.9,127.8,127.6,127.5$, $127.4,127.3,127.2,118.5,99.2,96.8,84.5,83.5,82.7$, 81.7, 79.7, 78.4, 75.5, 75.1, 74.4, 73, 71.2, 69.4, 68.9, 61, 60, 59.9, 58.1, 54.0; FABMS: $\mathrm{m} / \mathrm{z}$ calcd for $\mathrm{C}_{46} \mathrm{H}_{56} \mathrm{O}_{11}[\mathrm{M}+\mathrm{H}]^{+}$: 785.3901. Found: 785.3905.

3.14. $O$-(4,5,6,8-Tetra- $O$-benzyl-1,2-dideoxy- $\alpha$-D-glucooct-3-ulo-1-enopyranosyl)- $\mathrm{N}$-tert-butoxycarbonyl-Lserine methyl ester (12)

Yield: $73 \%$ as a colorless syrup; $[\alpha]_{\mathrm{D}}+54\left(c 1.4, \mathrm{CHCl}_{3}\right)$; ${ }^{1} \mathrm{H}$ NMR $\left(\mathrm{CDCl}_{3}, 400 \mathrm{MHz}\right): \delta 7.35-7.19(20 \mathrm{H}, \mathrm{m}$, $\mathrm{ArH}), 5.82\left(1 \mathrm{H}, \mathrm{dd}, J_{1^{\prime}, 2 \mathrm{a}^{\prime}}=11.6 \mathrm{~Hz}, J_{1^{\prime}, 2 \mathrm{~b}^{\prime}}=17.6 \mathrm{~Hz}\right.$, $\left.\mathrm{H} 1^{\prime}\right), 5.55\left(1 \mathrm{H}, \mathrm{dd}, J_{2 \mathrm{~b}^{\prime}, 2 \mathrm{a}^{\prime}}=1.6 \mathrm{~Hz}, J_{2 \mathrm{~b}^{\prime}, 1^{\prime}}=17.6 \mathrm{~Hz}\right.$, $\left.\mathrm{H} 2 \mathrm{~b}^{\prime}\right), 5.42(1 \mathrm{H}, \mathrm{d}, J=8.0 \mathrm{~Hz}, \mathrm{NH}), 5.33(1 \mathrm{H}, \mathrm{dd}$, $\left.J_{2 \mathrm{a}^{\prime}, 2 \mathrm{~b}^{\prime}}=1.6 \mathrm{~Hz}, \quad J_{2 \mathrm{a}^{\prime}, 1^{\prime}}=10.8 \mathrm{~Hz}, \quad \mathrm{H} 2 \mathrm{a}^{\prime}\right), \quad 4.85-4.79$ 
$\left(4 \mathrm{H}, \mathrm{m}, \mathrm{CH}_{2} \mathrm{Ph} \times 4\right), 4.65-4.52\left(4 \mathrm{H}, \mathrm{m}, \mathrm{CH}_{2} \mathrm{Ph} \times 4\right), 4.45$ $\left(1 \mathrm{H}, \mathrm{d}, J=8.4 \mathrm{~Hz}, \mathrm{H} 2^{\prime \prime}\right), 4.02\left(1 \mathrm{H}, \mathrm{dd}, J_{3,2}=8.8 \mathrm{~Hz}\right.$, $\left.J_{3,4}=9.2 \mathrm{~Hz}, \mathrm{H} 3\right), \quad 3.85\left(1 \mathrm{H}, \quad \mathrm{dd}, \quad J_{1 \mathrm{a}^{\prime \prime}, 2^{\prime \prime}}=2.4 \mathrm{~Hz}\right.$, $\left.J_{1 \mathrm{a}^{\prime \prime}, 1 \mathrm{~b}^{\prime \prime}}=10.0 \mathrm{~Hz}, \mathrm{H} 1 \mathrm{a}^{\prime \prime}\right), 3.77(1 \mathrm{H}, \mathrm{m}, \mathrm{H} 6), 3.70-3.66$ $(3 \mathrm{H}, \mathrm{m}, \mathrm{H} 4, \mathrm{H} 5, \mathrm{H} 6), 3.64\left(3 \mathrm{H}, \mathrm{s}, \mathrm{COOCH}_{3}\right), 3.55$ $\left(1 \mathrm{H}, \mathrm{dd}, J_{1 \mathrm{~b}^{\prime \prime}, 2^{\prime \prime}}=3.2 \mathrm{~Hz}, J_{1 \mathrm{~b}^{\prime \prime}, 1 \mathrm{a}^{\prime \prime}}=10 \mathrm{~Hz}, \mathrm{H} \mathrm{b}^{\prime \prime}\right), 3.32$ $\left(1 \mathrm{H}, \mathrm{d}, J_{2,3}=9.6 \mathrm{~Hz}, \mathrm{H} 2\right), 1.42\left(9 \mathrm{H}, \mathrm{s}, 3 \mathrm{CH}_{3}\right) ;{ }^{13} \mathrm{C}$ NMR $\left(\mathrm{CDCl}_{3}, 100 \mathrm{MHz}\right): \delta 171.1,155.6,138.9,138.7$, $138.5,134.9,128.7,128.5,128.4,128.2,128.1,128$, $127.9,127.8,127.7,127.6,127.5,127.1,119.5,99.6$, 84.6, 82.8, 78.4, 75.6, 75.4, 75.1, 73.6, 72.2, 68.8, 62.4, 54.0, 52.5, 28.5; FABMS: $m / z$ calcd for $\mathrm{C}_{45} \mathrm{H}_{53} \mathrm{O}_{10} \mathrm{Na}$ $[\mathrm{M}+\mathrm{Na}]^{+}:$790.3576. Found: 790.3553 .

\subsection{5. (Z)-3,7-Anhydro-4,5,6,8-tetra-O-benzyl-1-O-n- butoxycarbonyl-2,3-dideoxy-D-gluco-oct-2-enitol (13)}

To a solution of 17 (630 mg, $1.11 \mathrm{mmol})$ in dry $\mathrm{CH}_{2} \mathrm{Cl}_{2}$ $(10 \mathrm{~mL})$ was added butyl chloroformate $(2.22 \mathrm{~mL}$, $2.22 \mathrm{mmol})$ and pyridine $(447 \mu \mathrm{L}, 5.55 \mathrm{mmol})$ under Ar atmosphere at rt. After $20 \mathrm{~min}$, the reaction mixture was concentrated under reduced pressure to give a residue that was purified by silica gel chromatography (1:6, EtOAc/hexanes) to give $13(628 \mathrm{mg}, 85 \%)$ as a colorless syrup; $R_{\mathrm{f}} 0.27 ;[\alpha]_{\mathrm{D}}+40.4\left(c 2.6, \mathrm{CH}_{2} \mathrm{Cl}_{2}\right) ;{ }^{1} \mathrm{H} \mathrm{NMR}$ $\left(\mathrm{CD}_{2} \mathrm{Cl}_{2}, 500 \mathrm{MHz}\right): \delta$ 7.37-7.21 $(20 \mathrm{H}, \mathrm{m}, \mathrm{ArH}), 5.17$ $\left(1 \mathrm{H}, \quad \mathrm{t}, \quad J_{1^{\prime}, 2^{\prime}}=7.0 \mathrm{~Hz}, \quad \mathrm{H}^{\prime}\right), \quad 4.86 \quad(1 \mathrm{H}, \quad \mathrm{dd}$, $\left.J_{2 \mathrm{a}^{\prime}, 1^{\prime}}=7.5 \mathrm{~Hz}, J_{2 \mathrm{a}^{\prime}, 2 \mathrm{~b}^{\prime}}=12.0 \mathrm{~Hz}, \mathrm{H} 2 \mathrm{a}^{\prime}\right), 4.79(1 \mathrm{H}, \mathrm{d}$, $\left.J_{\mathrm{a}, \mathrm{b}}=12.5 \mathrm{~Hz}, \mathrm{CH}_{2} \mathrm{Ph}\right), 4.77-4.71\left(3 \mathrm{H}, \mathrm{m}, \mathrm{CH}_{2} \mathrm{Ph} \times 2\right.$, $\left.\mathrm{H} 2^{\prime}\right), 4.69\left(1 \mathrm{H}, \mathrm{d}, J_{\mathrm{a}, \mathrm{b}}=11 \mathrm{~Hz}, \mathrm{CH}_{2} \mathrm{Ph}\right), 4.63(1 \mathrm{H}, \mathrm{d}$, $\left.J_{\mathrm{a}, \mathrm{b}}=12.5 \mathrm{~Hz}, \mathrm{CH}_{2} \mathrm{Ph}\right), 4.61\left(1 \mathrm{H}, \mathrm{d}, J_{\mathrm{a}, \mathrm{b}}=12.0 \mathrm{~Hz}\right.$, $\left.\mathrm{CH}_{2} \mathrm{Ph}\right), 4.57\left(1 \mathrm{H}, \mathrm{d}, J_{\mathrm{a}, \mathrm{b}}=11.5 \mathrm{~Hz}, \mathrm{CH}_{2} \mathrm{Ph}\right), 4.57$ $\left(1 \mathrm{H}, \quad \mathrm{d}, \quad J_{\mathrm{a}, \mathrm{b}}=12.0 \mathrm{~Hz}, \quad \mathrm{CH}_{2} \mathrm{Ph}\right), \quad 4.13 \quad(2 \mathrm{H}, \quad \mathrm{t}$, $\left.J_{3^{\prime}, 4^{\prime}}=7.0 \mathrm{~Hz}, \mathrm{H}^{\prime}\right), 3.97\left(1 \mathrm{H}, \mathrm{d}, J_{2,3}=6.0 \mathrm{~Hz}, \mathrm{H} 2\right)$, $3.93\left(1 \mathrm{H}, \quad \mathrm{ddd}, \quad J_{5,4}=2.0 \mathrm{~Hz}, \quad J_{5,6 \mathrm{a}}=4.0 \mathrm{~Hz}\right.$, $\left.J_{5,6 \mathrm{~b}}=9.5 \mathrm{~Hz}, \quad \mathrm{H} 5\right), \quad 3.83\left(1 \mathrm{H}, \quad \mathrm{dd}, \quad J_{6 \mathrm{a}, 5}=1.5 \mathrm{~Hz}\right.$, $\left.J_{6 \mathrm{a}, 6 \mathrm{~b}}=11.0 \mathrm{~Hz}, \mathrm{H} 6 \mathrm{a}\right), 3.81-3.73(3 \mathrm{H}, \mathrm{m}, \mathrm{H} 3, \mathrm{H} 4$, H6), $1.66\left(2 \mathrm{H}, \mathrm{m}, \mathrm{H} 4 \mathrm{a}^{\prime}, \mathrm{H} 4 \mathrm{~b}^{\prime}\right), 1.41\left(2 \mathrm{H}, \mathrm{m}, \mathrm{H} 5 \mathrm{a}^{\prime}\right.$, $\left.\mathrm{H}_{5 b^{\prime}}\right), 0.95\left(3 \mathrm{H}, \mathrm{t}, J_{6^{\prime}, 5^{\prime}}=7.5 \mathrm{~Hz}, \mathrm{H}^{\prime} \times 3\right) ;{ }^{13} \mathrm{C} \mathrm{NMR}$ $\left(\mathrm{CD}_{2} \mathrm{Cl}_{2}, 125 \mathrm{MHz}\right): \delta$ 155.6, 153.2, 138.7, 138.2, $128.7,128.6,128.5,128.3,128.2,128.1,128,127.9$, 104.1, 84.3, 78.7, 78.2, 77.9, 77.8, 74.3, 74.0, 73.7, 72.6, 69.2, 68.0, 61.8, 31.1, 19.3, 13.8; FABMS: $m / z$ calcd for $\mathrm{C}_{41} \mathrm{H}_{46} \mathrm{O}_{8}[\mathrm{M}+\mathrm{H}]^{+}$: 666.3193 . Found: 666.3186 .

3.16. (Z)-3,7-Anhydro-4,5,6,8-tetra- $O$-benzyl-1- $O$-n-hexoxycarbonyl-2,3-dideoxy-D-gluco-oct-2-enitol (14)

To a stirred solution of $17(154 \mathrm{mg}, 0.27 \mathrm{mmol})$ in dry DMF $(5 \mathrm{~mL})$ were added 1,1'-carbonyl- $n$-hexanoxyimidazole (106 mg, $0.54 \mathrm{mmol}$ ) and $\mathrm{NaH}$ (21.6 mg, $0.54 \mathrm{mmol}$ ) under an argon atmosphere at rt. After $1 \mathrm{~h}, \mathrm{H}_{2} \mathrm{O}$ was added to quench the reaction and the solvent was removed under reduced pressure. The crude product was extracted by $\mathrm{CH}_{2} \mathrm{Cl}_{2}$ and the combined organic phase was dried $\left(\mathrm{MgSO}_{4}\right)$, concentrated, and purified by silica gel chromatography (1:5, EtOAc/hexanes) to give $14(183 \mathrm{mg}, 98 \%)$ as a colorless syrup; $R_{\mathrm{f}}$ 0.33 (1:5, EtOAc/hexanes); $[\alpha]_{\mathrm{D}}+36.7$ (c 3.1, $\mathrm{CHCl}_{3}$ ); ${ }^{1} \mathrm{H} \mathrm{NMR}\left(\mathrm{CDCl}_{3}, 400 \mathrm{MHz}\right): \delta 7.35-7.13(20 \mathrm{H}, \mathrm{m}$, $\mathrm{ArH}), 5.22\left(1 \mathrm{H}, \mathrm{dd}, J_{1^{\prime}, 2 \mathrm{a}^{\prime}}=6.8 \mathrm{~Hz}, J_{1^{\prime}, 2 \mathrm{~b}^{\prime}}=6.8 \mathrm{~Hz}\right.$, $\left.\mathrm{H} 1^{\prime}\right), 4.91\left(1 \mathrm{H}, \mathrm{dd}, J_{2 \mathrm{a}^{\prime}, 1^{\prime}}=6.8 \mathrm{~Hz}, J_{2 \mathrm{a}^{\prime}, 2 \mathrm{~b}^{\prime}}=12 \mathrm{~Hz}\right.$, $\left.\mathrm{H} 2 \mathrm{a}^{\prime}\right), 4.81\left(1 \mathrm{H}, \mathrm{d}, J_{\mathrm{a}, \mathrm{b}}=11.2 \mathrm{~Hz}, \mathrm{CH}_{2} \mathrm{Ph}\right), 4.76(1 \mathrm{H}$, $\left.\mathrm{d}, J_{\mathrm{a}, \mathrm{b}}=11.2 \mathrm{~Hz}, \mathrm{CH}_{2} \mathrm{Ph}\right), 4.73\left(1 \mathrm{H}, \mathrm{d}, J_{\mathrm{a}, \mathrm{b}}=11.6 \mathrm{~Hz}\right.$, $\left.\mathrm{CH}_{2} \mathrm{Ph}\right), \quad 4.72-4.70\left(1 \mathrm{H}, \mathrm{m}, \mathrm{H} 2^{\prime}\right), 4.69(1 \mathrm{H}, \mathrm{d}$, $\left.J_{\mathrm{a}, \mathrm{b}}=11.2 \mathrm{~Hz}, \mathrm{CH}_{2} \mathrm{Ph}\right), 4.64\left(1 \mathrm{H}, \mathrm{d}, J_{\mathrm{a}, \mathrm{b}}=12.0 \mathrm{~Hz}\right.$, $\left.\mathrm{CH}_{2} \mathrm{Ph}\right), 4.61\left(1 \mathrm{H}, \mathrm{d}, \mathrm{J}_{\mathrm{a}, \mathrm{b}}=11.6 \mathrm{~Hz}, \mathrm{CH}_{2} \mathrm{Ph}\right), 4.52$ $\left(1 \mathrm{H}, \quad \mathrm{d}, \quad J_{\mathrm{a}, \mathrm{b}}=12.0 \mathrm{~Hz}, \quad \mathrm{CH}_{2} \mathrm{Ph}\right), 4.51 \quad(1 \mathrm{H}, \mathrm{d}$, $\left.J_{\mathrm{a}, \mathrm{b}}=10.8 \mathrm{~Hz}, \quad \mathrm{CH}_{2} \mathrm{Ph}\right), \quad 4.11 \quad(2 \mathrm{H}, \quad \mathrm{d}, \quad J=6.8 \mathrm{~Hz}$, $\left.\mathrm{H}^{3} \mathrm{a}^{\prime}, 3 \mathrm{~b}^{\prime}\right), 3.94\left(1 \mathrm{H}, \mathrm{d}, J_{2,3}=6.8 \mathrm{~Hz}, \mathrm{H} 2\right), 3.84-3.68$ $(5 \mathrm{H}, \mathrm{m}, \mathrm{H} 3, \mathrm{H} 4, \mathrm{H} 5, \mathrm{H} 6 \mathrm{a}, \mathrm{b}), 1.68-1.61$ (2H, m, $\left.\mathrm{H}_{4} \mathrm{a}^{\prime}, \mathrm{b}^{\prime}\right), \quad 1.39-1.25\left(6 \mathrm{H}, \mathrm{H} 5 \mathrm{a}^{\prime}, \mathrm{b}^{\prime}, \mathrm{H} 6 \mathrm{a}^{\prime}, \mathrm{b}^{\prime}, \mathrm{H} 7 \mathrm{a}^{\prime}, \mathrm{b}^{\prime}\right)$, $0.88\left(3 \mathrm{H}, \mathrm{t}, J_{8^{\prime}, 7^{\prime}}=6.8 \mathrm{~Hz}, \mathrm{H}^{\prime} \times 3\right) ;{ }^{13} \mathrm{C} \mathrm{NMR}\left(\mathrm{CDCl}_{3}\right.$, $100 \mathrm{MHz}): \delta 55.5,153.3,138.3,138.2,138.1,137.8$, $128.6,128.5,128.1,128,127.9,127.8,104.1,84.7,78.8$, $78.5,77.4,74.6,74.5,73.7,73,68.6,68.3,61.8,31.6$, $28.8,25.5,22.7,14.2$; FABMS: $m / z$ calcd for $\mathrm{C}_{43} \mathrm{H}_{50} \mathrm{O}_{8}[\mathrm{M}+\mathrm{H}]^{+}$: 694.3506. Found: 694.3497.

\subsection{7. ( $Z$ )-3,7-Anhydro-4,5,6,8-tetra- $O$-benzyl-1- $O$-benz- yloxycarbonyl-2,3-dideoxy-D-gluco-oct-2-enitol (15)}

The preparation was similar to that of compound $\mathbf{1 3}$. Yield: $82 \%$ as a colorless syrup; $[\alpha]_{\mathrm{D}}+44$ (c 6.1, $\left.\mathrm{CH}_{2} \mathrm{Cl}_{2}\right) ;{ }^{1} \mathrm{H} \mathrm{NMR}\left(\mathrm{CD}_{2} \mathrm{Cl}_{2}, 500 \mathrm{MHz}\right): \delta$ 7.38-7.18 $(25 \mathrm{H}, \mathrm{m}, \mathrm{ArH}), 5.31-5.30\left(2 \mathrm{H}, \mathrm{m}, \mathrm{CH}_{2} \mathrm{Ph} \times 2\right), 5.15-$ $5.12\left(1 \mathrm{H}, \mathrm{m}, \mathrm{H} 1^{\prime}\right), 4.85\left(1 \mathrm{H}, \mathrm{dd}, J_{2 \mathrm{a}^{\prime}, 1^{\prime}}=7.5 \mathrm{~Hz}\right.$, $\left.J_{2 \mathrm{a}^{\prime}, 2 \mathrm{~b}^{\prime}}=12 \mathrm{~Hz}, \mathrm{H} 2 \mathrm{a}^{\prime}\right), 4.75\left(1 \mathrm{H}, \mathrm{d}, J_{\mathrm{a}, \mathrm{b}}=11.5 \mathrm{~Hz}\right.$, $\left.\mathrm{CH}_{2} \mathrm{Ph}\right), \quad 4.73-4.70 \quad\left(1 \mathrm{H}, \mathrm{m}, \mathrm{H} 2^{\prime}\right), 4.70 \quad(2 \mathrm{H}, \mathrm{d}$, $\left.J_{\mathrm{a}, \mathrm{b}}=12.0 \mathrm{~Hz}, \mathrm{CH}_{2} \mathrm{Ph}\right), 4.65\left(1 \mathrm{H}, \mathrm{d}, J_{\mathrm{a}, \mathrm{b}}=11.5 \mathrm{~Hz}\right.$, $\left.\mathrm{CH}_{2} \mathrm{Ph}\right), 4.59\left(1 \mathrm{H}, \mathrm{d}, J_{\mathrm{a}, \mathrm{b}}=12.0 \mathrm{~Hz}, \mathrm{CH}_{2} \mathrm{Ph}\right), 4.56$ $\left(1 \mathrm{H}, \quad \mathrm{d}, \quad J_{\mathrm{a}, \mathrm{b}}=11.5 \mathrm{~Hz}, \quad \mathrm{CH}_{2} \mathrm{Ph}\right), 4.53 \quad(1 \mathrm{H}, \mathrm{d}$, $\left.J_{\mathrm{a}, \mathrm{b}}=11.0 \mathrm{~Hz}, \quad \mathrm{CH}_{2} \mathrm{Ph}\right), \quad 4.52 \quad\left(1 \mathrm{H}, \quad J_{\mathrm{a}, \mathrm{b}}=12.0 \mathrm{~Hz}\right.$, $\left.\mathrm{CH}_{2} \mathrm{Ph}\right), 3.93\left(1 \mathrm{H}, \mathrm{d}, J_{2,3}=6.0 \mathrm{~Hz}, \mathrm{H} 2\right), 3.88(1 \mathrm{H}$, ddd, $\left.J_{5,4}=2.0 \mathrm{~Hz}, J_{5,6 \mathrm{a}}=4.0 \mathrm{~Hz}, J_{5,6 \mathrm{~b}}=10.0 \mathrm{~Hz}, \mathrm{H} 5\right)$, $3.78\left(1 \mathrm{H}, \mathrm{dd}, J_{6 \mathrm{a}, 5}=4.0 \mathrm{~Hz}, J_{6 \mathrm{a}, 6 \mathrm{~b}}=12.0 \mathrm{~Hz}\right.$, H6a), 3.76-3.71 (2H, m, H4, H6b), 3.70 (1H, dd, $\left.J_{3,2}=6.5 \mathrm{~Hz}, J_{3,4}=6.5 \mathrm{~Hz}, \mathrm{H} 3\right) ;{ }^{13} \mathrm{C} \mathrm{NMR}\left(\mathrm{CD}_{2} \mathrm{Cl}_{2}\right.$, $100 \mathrm{MHz}): \delta 155.4,153.6,138.7,138.6,138.2,128.8$, $128.7,128.6,128.5,128.3,128.2,128.1,128,127.9$, 103.9, 84.2, 78.6, 78.2, 77.8, 74.3, 74, 73.7, 72.5, 69.7, 69.2, 62.1; FABMS: $m / z$ calcd for $\mathrm{C}_{44} \mathrm{H}_{44} \mathrm{O}_{8} \mathrm{Na}$ $[\mathrm{M}+\mathrm{Na}]^{+}:$723.2934. Found: 723.2919.

\subsection{8. (Z)-3,7-Anhydro-4,5,6,8-tetra- $O$-benzyl-2,3-di- deoxy-1- $O$-[(methyl 2,3,4-tri- $O$-methyl- $\alpha$-D-glucopyran- oside-6- $O$-yl)carbonyl|-D-gluco-oct-2-enitol (16)}

To a stirred solution of $17(128 \mathrm{mg}, 0.27 \mathrm{mmol})$ in dry DMF (5 mL) were added $18(112 \mathrm{mg}, 0.34 \mathrm{mmol})$ and $\mathrm{NaH}$ (21.6 mg, $0.54 \mathrm{mmol})$ under an argon atmosphere at rt. After $1 \mathrm{~h}$, the reaction was quenched by the 
addition of water and then concentrated under reduced pressure. The crude product was extracted by $\mathrm{CH}_{2} \mathrm{Cl}_{2}$ and the combined organic phase was dried $\left(\mathrm{MgSO}_{4}\right)$, concentrated, and purified by silica gel chromatography (1:1, EtOAc/hexanes) to give $\mathbf{1 6}(185 \mathrm{mg}, 88 \%)$ as a colorless syrup; $R_{\mathrm{f}} 0.4$ (1:1, EtOAc/hexanes); $[\alpha]_{\mathrm{D}}+92$ (c 1.0, $\left.\mathrm{CHCl}_{3}\right) ;{ }^{1} \mathrm{H}$ NMR $\left(\mathrm{CDCl}_{3}, 500 \mathrm{MHz}\right): \delta 7.32$ $7.12(20 \mathrm{H}, \mathrm{m}, \mathrm{ArH}), 5.19\left(1 \mathrm{H}, \mathrm{dd}, J_{1^{\prime}, 2 \mathrm{a}^{\prime}}=7.0 \mathrm{~Hz}\right.$, $\left.J_{1^{\prime}, 2 \mathrm{~b}^{\prime}}=7.5 \mathrm{~Hz}, \mathrm{H} 1^{\prime}\right), 4.92\left(1 \mathrm{H}, \mathrm{dd}, J_{2 \mathrm{~b}^{\prime}, 1^{\prime}}=8.0 \mathrm{~Hz}\right.$, $\left.J_{2 \mathrm{~b}^{\prime}, 2 \mathrm{a}^{\prime}}=12.5 \mathrm{~Hz}, \mathrm{H} 2 \mathrm{~b}^{\prime}\right), 4.78\left(1 \mathrm{H}, \mathrm{d}, J_{\mathrm{a}, \mathrm{b}}=12.0 \mathrm{~Hz}\right.$, $\left.\mathrm{CH}_{2} \mathrm{Ph}\right), 4.77\left(1 \mathrm{H}, \mathrm{d}, J_{3^{\prime}, 4^{\prime}}=4.0 \mathrm{~Hz}, \mathrm{H} 3^{\prime}\right), 4.74(1 \mathrm{H}, \mathrm{d}$, $\left.J_{\mathrm{a}, \mathrm{b}}=11.0 \mathrm{~Hz}, \mathrm{CH}_{2} \mathrm{Ph}\right), 4.70\left(1 \mathrm{H}, \mathrm{d}, J_{\mathrm{a}, \mathrm{b}}=11.5 \mathrm{~Hz}\right.$, $\left.\mathrm{CH}_{2} \mathrm{Ph}\right), 4.71\left(1 \mathrm{H}, \mathrm{m}, \mathrm{H} 2^{\prime}\right), 4.67\left(1 \mathrm{H}, \mathrm{d}, J_{\mathrm{a}, \mathrm{b}}=11.0 \mathrm{~Hz}\right.$, $\left.\mathrm{CH}_{2} \mathrm{Ph}\right), 4.62\left(1 \mathrm{H}, \mathrm{d}, J_{\mathrm{a}, \mathrm{b}}=12.0 \mathrm{~Hz}, \mathrm{CH}_{2} \mathrm{Ph}\right), 4.59(1 \mathrm{H}$, $\left.\mathrm{d}, J_{\mathrm{a}, \mathrm{b}}=11.5 \mathrm{~Hz}, \mathrm{CH}_{2} \mathrm{Ph}\right), 4.51\left(1 \mathrm{H}, \mathrm{d}, J_{\mathrm{a}, \mathrm{b}}=12.0 \mathrm{~Hz}\right.$, $\left.\mathrm{CH}_{2} \mathrm{Ph}\right), 4.50\left(1 \mathrm{H}, \mathrm{d}, J_{\mathrm{a}, \mathrm{b}}=11.0 \mathrm{~Hz}, \mathrm{CH}_{2} \mathrm{Ph}\right), 4.35$ $\left(1 \mathrm{H}, \mathrm{dd}, J_{6 \mathrm{a}, 5}=2 \mathrm{~Hz}, J_{6 \mathrm{a}, 6 \mathrm{~b}}=11.5 \mathrm{~Hz}, \mathrm{H} 6 \mathrm{a}\right), 4.27(1 \mathrm{H}$, $\left.\mathrm{dd}, J_{6 \mathrm{~b}, 5}=5.0 \mathrm{~Hz}, J_{6 \mathrm{~b}, 6 \mathrm{a}}=12.0 \mathrm{~Hz}, \mathrm{H} 6 \mathrm{~b}\right), 3.92(1 \mathrm{H}, \mathrm{d}$, $\left.J_{2,3}=7.0 \mathrm{~Hz}, \mathrm{H} 2\right), 3.8-3.72\left(4 \mathrm{H}, \mathrm{m}, \mathrm{H} 4, \mathrm{H} 5, \mathrm{H}^{\prime} \mathrm{a}^{\prime}\right.$, $\left.8 \mathrm{~b}^{\prime}\right), 3.70-3.65\left(2 \mathrm{H}, \mathrm{m}, \mathrm{H} 3, \mathrm{H}^{\prime}\right), 3.59\left(3 \mathrm{H}, \mathrm{s}, \mathrm{OCH}_{3}\right)$, $3.51\left(3 \mathrm{H}, \mathrm{s}, \mathrm{OCH}_{3}\right), 3.49\left(1 \mathrm{H}, \mathrm{m}, \mathrm{H} 5^{\prime}\right), 3.47(1 \mathrm{H}, \mathrm{s}$, $\left.\mathrm{OCH}_{3}\right), \quad 3.37\left(1 \mathrm{H}, \quad \mathrm{s}, \quad \mathrm{OCH}_{3}\right), \quad 3.17 \quad(1 \mathrm{H}, \quad \mathrm{dd}$, $\left.J_{4^{\prime}, 3^{\prime}}=4.0 \mathrm{~Hz}, J_{4^{\prime}, 5^{\prime}}=10.0 \mathrm{~Hz}, \mathrm{H}^{\prime}\right), 3.08(1 \mathrm{H}, \mathrm{dd}$, $\left.J_{6^{\prime}, 7^{\prime}}=9.0 \mathrm{~Hz}, \quad J_{6^{\prime}, 5^{\prime}}=10.0 \mathrm{~Hz}, \quad \mathrm{H} 6^{\prime}\right) ; \quad{ }^{13} \mathrm{C} \quad \mathrm{NMR}$ $\left(\mathrm{CD}_{2} \mathrm{Cl}_{2}, 125 \mathrm{MHz}\right): \delta \quad 155.3,153.4,138.3,138.2$, $138.1,137.7,128.6,128.5,128.1,128,127.9,103.9$, $97.5,84.7,83.6,81.8,79.6,78.8,78.5,76.9,74.6,74.5$, $73.7,68.7,68.6,66.5,62.1,61.1,60.8,59.2,55.4$; FABMS: $m / z$ calcd for $\mathrm{C}_{47} \mathrm{H}_{56} \mathrm{O}_{13} \mathrm{Na}[\mathrm{M}+\mathrm{Na}]^{+}$: 851.3619. Found: 851.3634.

\subsection{9. $n$-Hexyl 4,5,6,8-tetra- $O$-benzyl-1,2-dideoxy- $\alpha$-D- gluco-oct-3-ulo-1-enopyranoside (19)}

A stirred solution of $14(52 \mathrm{mg}, 0.075 \mathrm{mmol})$ in dry 1nitropropane $(2 \mathrm{~mL})$ was heated at $125^{\circ} \mathrm{C}$ for $4 \mathrm{~h}$. The solvent was then removed under reduced pressure yielding a residue that was purified by silica gel chromatography $(1: 10$, EtOAc/hexanes) to give $\mathbf{1 9}(9.7 \mathrm{mg}, 20 \%)$ as a colorless syrup; $R_{\mathrm{f}} 0.2$ (1:10, EtOAc/hexanes); $[\alpha]_{\mathrm{D}}+41$ (c 1.4, $\left.\mathrm{CHCl}_{3}\right) ;{ }^{1} \mathrm{H}$ NMR $\left(\mathrm{CD}_{2} \mathrm{Cl}_{2}, 400 \mathrm{MHz}\right): \delta 7.41-$ $7.27(20 \mathrm{H}, \mathrm{m}, \mathrm{ArH}), 6.01\left(1 \mathrm{H}, \mathrm{dd}, J_{1^{\prime}, 2 \mathrm{a}^{\prime}}=11.2 \mathrm{~Hz}\right.$, $\left.J_{1^{\prime}, 2 \mathrm{~b}^{\prime}}=17.6 \mathrm{~Hz}, \mathrm{H} 1^{\prime}\right), 5.60\left(1 \mathrm{H}, \mathrm{dd}, J_{2 \mathrm{~b}^{\prime}, 2 \mathrm{a}^{\prime}}=2 \mathrm{~Hz}\right.$, $\left.J_{2 \mathrm{~b}^{\prime}, 1^{\prime}}=17.6 \mathrm{~Hz}, \mathrm{H} 2 \mathrm{~b}^{\prime}\right), 5.40\left(1 \mathrm{H}, \mathrm{m}, \mathrm{H} 2 \mathrm{a}^{\prime}\right), 4.96(1 \mathrm{H}$, $\left.\mathrm{d}, J_{\mathrm{a}, \mathrm{b}}=11.2 \mathrm{~Hz}, \mathrm{CH}_{2} \mathrm{Ph}\right), 4.91\left(1 \mathrm{H}, \mathrm{d}, J_{\mathrm{a}, \mathrm{b}}=11.2 \mathrm{~Hz}\right.$, $\left.\mathrm{CH}_{2} \mathrm{Ph}\right), 4.9\left(1 \mathrm{H}, \mathrm{d}, J_{\mathrm{a}, \mathrm{b}}=11.2 \mathrm{~Hz}, \mathrm{CH}_{2} \mathrm{Ph}\right), 4.85(1 \mathrm{H}$, $\left.\mathrm{d}, J_{\mathrm{a}, \mathrm{b}}=10.8 \mathrm{~Hz}, \mathrm{CH}_{2} \mathrm{Ph}\right), 4.68\left(1 \mathrm{H}, \mathrm{d}, J_{\mathrm{a}, \mathrm{b}}=12.4 \mathrm{~Hz}\right.$, $\left.\mathrm{CH}_{2} \mathrm{Ph}\right), 4.66\left(1 \mathrm{H}, \mathrm{d}, J_{\mathrm{a}, \mathrm{b}}=10.8 \mathrm{~Hz}, \mathrm{CH}_{2} \mathrm{Ph}\right), 4.65$ $\left(1 \mathrm{H}, \quad \mathrm{d}, \quad J_{\mathrm{a}, \mathrm{b}}=11.6 \mathrm{~Hz}, \quad \mathrm{CH}_{2} \mathrm{Ph}\right), \quad 4.62(1 \mathrm{H}, \quad \mathrm{d}$, $\left.J_{\mathrm{a}, \mathrm{b}}=12 \mathrm{~Hz}, \quad \mathrm{CH}_{2} \mathrm{Ph}\right), 4.10 \quad\left(1 \mathrm{H}, \mathrm{dd}, J_{3,2}=8.8 \mathrm{~Hz}\right.$, $\left.J_{3,4}=9.2 \mathrm{~Hz}, \mathrm{H} 3\right), 3.85-3.76(3 \mathrm{H}, \mathrm{m}, \mathrm{H} 5, \mathrm{H} 6 \mathrm{a}, \mathrm{b}), 3.68$ $\left(1 \mathrm{H}, \mathrm{dd}, J_{4,3}=9.2 \mathrm{~Hz}, \mathrm{H} 4\right), 3.45-3.34(3 \mathrm{H}, \mathrm{m}, \mathrm{H} 2$, H1a", $\left.\mathrm{b}^{\prime \prime}\right), 1.65-1.6\left(2 \mathrm{H}, \mathrm{m}, \mathrm{H} 2 \mathrm{a}^{\prime \prime}, \mathrm{b}^{\prime \prime}\right), 1.4-1.32(6 \mathrm{H}, \mathrm{m}$, $\left.\mathrm{H} 3 \mathrm{a}^{\prime \prime}, \mathrm{b}^{\prime \prime}, \mathrm{H} 4 \mathrm{a}^{\prime \prime}, \mathrm{b}^{\prime \prime}, \mathrm{H} 5 \mathrm{a}^{\prime \prime}, \mathrm{b}^{\prime \prime}\right), 0.93\left(3 \mathrm{H}, \mathrm{t}, J_{6^{\prime \prime}, 5^{\prime \prime}}=6.8 \mathrm{~Hz}\right.$, $\left.\mathrm{H} 6{ }^{\prime \prime} \times 3\right) ;{ }^{13} \mathrm{C} \mathrm{NMR}\left(\mathrm{CDCl}_{3}, 100 \mathrm{MHz}\right): \delta 139,138.6$, $138.4,136,128.6,128.5,128.4,128.3,128.2,128.1$,
$128,127.9,127.8,127.7,118.5,84.7,83.2,78.7,77.4$, 75.8, 75.7, 75.2, 73.5, 71.9, 69.0, 62.0, 31.8, 29.8, 26.1, 22.8; FABMS: $m / z$ calcd for $\mathrm{C}_{42} \mathrm{H}_{50} \mathrm{O}_{6} \mathrm{Na}[\mathrm{M}+\mathrm{Na}]^{+}$: 673.3505. Found 673.3505.

\subsection{Benzyl 4,5,6,8-tetra- $O$-benzyl-1,2-dideoxy- $\alpha$-D- gluco-oct-3-ulo-1-enopyranoside (20)}

A stirred solution of $15(55 \mathrm{mg}, 0.078 \mathrm{mmol})$ in dry 1nitropropane $(2 \mathrm{~mL})$ was heated at $125^{\circ} \mathrm{C}$ for $2 \mathrm{~h}$. The solvent was then removed under reduced pressure yielding a residue that was purified by silica gel chromatography (1:10, EtOAc/hexanes) to give $\mathbf{2 0}(28 \mathrm{mg}, 55 \%)$ as a colorless syrup; $R_{\mathrm{f}} 0.24$ (1:10, EtOAc/hexanes); ${ }^{1} \mathrm{H}$ NMR $\left(\mathrm{CD}_{2} \mathrm{Cl}_{2}, 500 \mathrm{MHz}\right): \delta \quad 7.35-7.22(25 \mathrm{H}, \mathrm{m}$, $\mathrm{ArH}), 6.05\left(1 \mathrm{H}, \mathrm{dd}, J_{1^{\prime}, 2 \mathrm{a}^{\prime}}=11.0 \mathrm{~Hz}, J_{1^{\prime}, 2 \mathrm{~b}^{\prime}}=17.5 \mathrm{~Hz}\right.$, $\left.\mathrm{H}^{\prime}\right), 5.64\left(1 \mathrm{H}, \mathrm{dd}, J_{2 \mathrm{~b}^{\prime}, 2 \mathrm{a}^{\prime}}=1.5 \mathrm{~Hz}, J_{2 \mathrm{~b}^{\prime}, 1^{\prime}}=17.5 \mathrm{~Hz}\right.$, $\left.\mathrm{H} 2 \mathrm{~b}^{\prime}\right), 3.39\left(1 \mathrm{H}, \mathrm{dd}, J_{2 \mathrm{a}^{\prime}, 2 \mathrm{~b}^{\prime}}=1.5 \mathrm{~Hz}, J_{2 \mathrm{a}^{\prime}, 1^{\prime}}=11.0 \mathrm{~Hz}\right.$, $\left.\mathrm{H} 2 \mathrm{a}^{\prime}\right), 4.90\left(1 \mathrm{H}, \mathrm{d}, J_{\mathrm{a}, \mathrm{b}}=11.0 \mathrm{~Hz}, \mathrm{CH}_{2} \mathrm{Ph}\right), 4.86-4.83$ $\left(3 \mathrm{H}, \quad \mathrm{m}, \quad \mathrm{CH}_{2} \mathrm{Ph} \times 3\right), 4.64\left(1 \mathrm{H}, \quad \mathrm{d}, \quad J_{\mathrm{a}, \mathrm{b}}=10.5 \mathrm{~Hz}\right.$, $\left.\mathrm{CH}_{2} \mathrm{Ph}\right), 4.63\left(1 \mathrm{H}, \mathrm{d}, J_{\mathrm{a}, \mathrm{b}}=12.0 \mathrm{~Hz}, \mathrm{CH}_{2} \mathrm{Ph}\right), 4.61$ $\left(1 \mathrm{H}, \quad \mathrm{d}, \quad J_{\mathrm{a}, \mathrm{b}}=11.0 \mathrm{~Hz}, \quad \mathrm{CH}_{2} \mathrm{Ph}\right), \quad 4.56 \quad(1 \mathrm{H}, \quad \mathrm{d}$, $\left.J_{\mathrm{a}, \mathrm{b}}=12.0 \mathrm{~Hz}, \mathrm{CH}_{2} \mathrm{Ph}\right), 4.48\left(1 \mathrm{H}, \mathrm{d}, J_{\mathrm{a}, \mathrm{b}}=12.0 \mathrm{~Hz}\right.$, $\left.\mathrm{CH}_{2} \mathrm{Ph}\right), 4.42\left(1 \mathrm{H}, \mathrm{d}, J_{\mathrm{a}, \mathrm{b}}=12.0 \mathrm{~Hz}, \mathrm{CH}_{2} \mathrm{Ph}\right), 4.09$ $\left(1 \mathrm{H}, \quad \mathrm{dd}, \quad J_{3,4}=9.0 \mathrm{~Hz}, J_{3,2}=9.5 \mathrm{~Hz}, \mathrm{H} 3\right), \quad 3.79-3.7$ $(3 \mathrm{H}, \mathrm{m}, \mathrm{H} 5, \mathrm{H} 6 \mathrm{a}, \mathrm{b}), 3.65\left(1 \mathrm{H}, \mathrm{dd}, J_{4,3}=9.0 \mathrm{~Hz}\right.$, $\left.J_{4,5}=9.5 \mathrm{~Hz}, \mathrm{H} 4\right), 3.39\left(1 \mathrm{H}, \mathrm{d}, J_{2,3}=9.5 \mathrm{~Hz}, \mathrm{H} 2\right) ;{ }^{13} \mathrm{C}$ NMR $\left(\mathrm{CD}_{2} \mathrm{Cl}_{2}, 125 \mathrm{MHz}\right): \delta 139.4,139,138.9,138.8$, $136,128.6,128.4,128.2,128,127.9,127.8,127.7$, $119.1,100.3,85.0,83.3,78.9,76.1,75.7,75.2,73.7$, 72.4, 69.4, 63.9; FABMS: $m / z$ calcd for $\mathrm{C}_{43} \mathrm{H}_{44} \mathrm{O}_{6}$ $[\mathrm{M}+\mathrm{H}]^{+}: 656.3138$.

\section{Acknowledgments}

The authors thank the financial support from the National Science Council of Taiwan (NSC93-2113-M001-003 and NSC93-2113-M-001-034) and Academia Sinica, Taiwan.

\section{References}

1. Bischofberger, K.; Hall, R. H.; Jordan, A. J. Chem. Soc., Chem. Commun. 1975, 806-807.

2. Yang, W.-B.; Chang, C.-F.; Wang, S.-H.; Teo, C.-F.; Lin, C.-H. Tetrahedron Lett. 2001, 42, 4657-4660.

3. Yang, W.-B.; Wu, C.-Y.; Chang, C.-C.; Wang, S.-H.; Teo, C.-F.; Lin, C.-H. Tetrahedron Lett. 2001, 42, 6907-6910.

4. Yang, W.-B.; Yang, Y.-Y.; Gu, Y.-F.; Wang, S.-H.; Chang, C.-C.; Lin, C.-H. J. Org. Chem. 2002, 67, 37733782.

5. For representative reviews, please see: (a) Tailefumier, C.; Chapleur, Y. Chem. Rev. 2004, 104, 263-292; (b) Lin, C.-H.; Lin, H.-C.; Yang, W.-B. Curr. Top. Med. Chem. 2005, 5, 1431-1457.

6. Chang, C.-F.; Yang, W.-B.; Chang, C.-C.; Lin, C.-H. Tetrahedron Lett. 2002, 43, 6515-6519. 
7. Lin, H.-C.; Yang, W.-B.; Gu, Y.-F.; Chen, C.-Y.; Wu, C.-Y.; Lin, C.-H. Org. Lett. 2003, 5, 1087-1089.

8. Lin, H.-C.; Chang, C.-C.; Chen, J.-Y.; Lin, C.-H. Tetrahedron: Asymmetry 2005, 16, 297-301.

9. Lin, H.-C.; Du, W.-P.; Chang, C.-C.; Lin, C.-H. Tetrahedron Lett. 2005, 46, 5071-5076.

10. March, J. In Advanced Organic Chemistry, 4th ed.; John Wiley \& Sons: New York, 1992; pp 356-362.

11. Maria, P.-C.; Gal, J.-F.; de Franceschi, J.; Fargin, E. J. Am. Chem. Soc. 1987, 109, 483-492.

12. Maria, P.-C.; Gal, J.-F. J. Phys. Chem. 1985, 89, 1296-1304.

13. (a) Tvaroska, I.; Taravel, F. R. Adv. Carbohydr. Chem. Biochem. 1995, 51, 15-61; (b) Li, X.; Ohtake, H.; Takahashi, H.; Ikegami, S. Tetrahedron 2001, 57, 4297-4309; (c) Li, X.; Takahashi, H.; Ohtake, H.; Shiro, M.; Ikegami, S. Tetrahedron 2001, 57, 8053-8066; (d) Schlesselmann, P.; Fritz, H.; Lehmann, J.; Uchiyama, T.; Brewer, C. F.; Hehre, E. J. Biochemistry 1982, 21, 6606-6614.

14. For an in-depth review, see: Panza, L.; Lay, L. In Carbohydrates in Chemistry and Biology; Ernst, B., Hart, G. W., Sinay, P., Eds.; Wiley-VCH Verlag GmbH: Weinheim, Germany, 2000; Vol. 1, pp 195-237.
15. Mukaiyama, T.; Sasaki, T.; Iwashita, E.; Matsubara, K. Chem. Lett. 1995, 455-456.

16. (a) Mukaiyama, T.; Miyazaki, K.; Uchiro, H. Chem. Lett. 1998, 635-636; (b) Mukaiyama, T.; Wakiyama, Y.; Miyazaki, K.; Takeuchi, K. Chem. Lett. 1999, 933-934.

17. Ford, M. J.; Ley, S. V. Synlett 1990, 255-256.

18. Ford, M. J.; Knight, J. G.; Ley, S. V.; Vile, S. Synlett 1990 , 331-332.

19. Rannard, S. P.; Davis, N. J. Org. Lett. 1999, 1, 933936.

20. (a) Ito, Y.; Ogawa, T. J. Am. Chem. Soc. 1997, 119, 55625566; (b) Matsuo, I.; Wada, M.; Manabe, S.; Yamaguguchi, Y.; Otake, K.; Kato, K.; Ito, Y. J. Am. Chem. Soc. 2003, 125, 3402-3403.

21. Iimori, T.; Shibasaki, T.; Ikegami, S. Tetrahedron Lett. 1996, 37, 2267-2270.

22. Iimori, T.; Azumaya, I.; Shibasaki, T.; Ikegami, S. Heterocycles 1997, 46, 221-224.

23. Azumaya, I.; Niwa, T.; Kotani, M.; Iimori, T.; Ikegami, S. Tetrahedron Lett. 1999, 40, 4683-4686.

24. Scheffler, G.; Schmidt, R. R. Tetrahedron Lett. 1997, 38, 2943-2946. 